TRANSACTIONS OF THE

AMERICAN MATHEMATICAL SOCIETY

Volume 352, Number 12, Pages 5855-5876

S 0002-9947(00)02639-8

Article electronically published on August 8, 2000

\title{
QUANTUM $n$-SPACE AS A QUOTIENT OF CLASSICAL $n$-SPACE
}

\author{
K. R. GOODEARL AND E. S. LETZTER
}

\begin{abstract}
The prime and primitive spectra of $\mathcal{O}_{\mathbf{q}}\left(k^{n}\right)$, the multiparameter quantized coordinate ring of affine $n$-space over an algebraically closed field $k$, are shown to be topological quotients of the corresponding classical spectra, $\operatorname{spec} \mathcal{O}\left(k^{n}\right)$ and $\max \mathcal{O}\left(k^{n}\right) \approx k^{n}$, provided the multiplicative group generated by the entries of $\mathbf{q}$ avoids -1 .
\end{abstract}

\section{INTRODUCTION}

In the representation theory of a noncommutative ring $A$, the natural analog of the maximal spectrum of a commutative ring is prim $A$, the set of primitive ideals (i.e., annihilators of irreducible $A$-modules). Moreover, $\operatorname{prim} A$ can be equipped with the Zariski topology (which in this setting is also called the Jacobson topology). Thus, if $A$ is a quantization of a classical coordinate $\operatorname{ring} \mathcal{O}(V)$ over an algebraically closed field, one can view $\operatorname{prim} A$ as a quantization of the variety $V$. The question then naturally arises: how are prim $A$ and $V$ related? Some "piecewise" relations are known in many cases. For instance, if $G$ is a connected, semisimple, complex algebraic group and $H$ is a maximal torus of $G$, then various (generic) quantizations $A$ of $\mathcal{O}(G)$ exhibit the following properties: $H$ acts on $A$ via automorphisms; there are only finitely many $H$-orbits in $\operatorname{prim} A$, and they are locally closed; each $H$ orbit in $\operatorname{prim} A$ is homeomorphic to a torus; and each $H$-orbit in $\operatorname{prim} A$ is a settheoretic quotient of a locally closed subset of $G$ that is stable under translation by $H$ (see [8], 9], [1], [12, [10]). Similar pictures have been observed to hold for quantized coordinate rings of affine spaces (see 4], 15], 3], 6]). One is therefore led to conjecture that in the above situations, $\operatorname{prim} A$ as a whole is a topological quotient of $V$. Similarly, $\operatorname{spec} A$ (see below) should be a topological quotient of $\operatorname{spec} \mathcal{O}(V)$. In the "smallest" cases, such as $A=\mathcal{O}_{q}\left(S L_{2}(k)\right)$ and $A=\mathcal{O}_{q}\left(k^{2}\right)$, these conjectures can easily be verified by direct calculation. In this paper we establish these conjectures for multiparameter quantum affine spaces, when -1 cannot be written as a product of the defining parameters.

To provide some detail, first let $k$ be an algebraically closed field. By a quantum $k$-affine $n$-space (i.e., a quantization of the coordinate ring of $k^{n}$ ), we mean an algebra with generators $x_{1}, x_{2}, \ldots, x_{n}$ subject only to the commutator relations $x_{i} x_{j}=q_{i j} x_{j} x_{i}$, for a chosen set of nonzero scalars $q_{i j} \in k$. (To avoid degeneracies,

Received by the editors April 22, 1999.

2000 Mathematics Subject Classification. Primary 16D60, 16P40, 16S36, 16W35; Secondary 20G42, 81R50.

The research of the first author was partially supported by NSF grant DMS-9622876, and the research of the second author was partially supported by NSF grant DMS-9623579. 
one assumes that $\mathbf{q}=\left(q_{i j}\right)$ is a multiplicatively antisymmetric $n \times n$ matrix. $)$ We denote this algebra as $\mathcal{O}_{\mathbf{q}}\left(k^{n}\right)$. Letting $H:=\left(k^{\times}\right)^{n}$ be an algebraic $n$-torus, it is not hard to verify that the natural action of $H$ on the vector space spanned by $x_{1}, \ldots, x_{n}$ extends to an action by $k$-algebra automorphisms on $\mathcal{O}_{\mathbf{q}}\left(k^{n}\right)$.

Next, set $A=\mathcal{O}_{\mathbf{q}}\left(k^{n}\right)$. The $H$-action on $A$ described in the previous paragraph extends naturally to actions on $\operatorname{prim} A$ and on the larger set $\operatorname{spec} A$ of prime ideals of $A$. (Recall that a ring is prime when every product of nonzero ideals is nonzero, and that an ideal $I$ of $A$ is prime when $A / I$ is a prime ring. It is important to note, for the prime ideals $I$ we are considering in this paper, that $A / I$ may have zero-divisors.) We can impose the Zariski topology on $\operatorname{spec} A$, and $\operatorname{prim} A$ then becomes a subspace under the relative topology.

Our methods now require us to make the following technical assumption: Either the subgroup of $k^{\times}$generated by the $q_{i j}$ does not contain -1 , or the characteristic of $k$ is 2 . (While our present techniques do not appear to work in the absence of this hypothesis, we do not know if our main results will remain valid without it.) Now let $R:=\mathcal{O}\left(k^{n}\right)$ be a commutative polynomial ring in $n$ variables, also equipped with the natural action of $H$ by automorphisms, and continue to let $A=\mathcal{O}_{\mathbf{q}}\left(k^{n}\right)$. In the main result $(4.11)$, we construct $H$-equivariant topological quotient maps

$$
\operatorname{spec} R \rightarrow \operatorname{spec} A \quad \text { and } \quad k^{n} \approx \max R \rightarrow \operatorname{prim} A
$$

Moreover, a description of the fibers of the second map is provided. Roughly speaking, we show that $\operatorname{prim} A$ is equal to $k^{n}$ modulo the actions of a compatible system of groups acting separately on each $H$-orbit, and that spec $A$ can be obtained from spec $R$ in an analogous fashion. These quotient maps are not uniquely determined by the $q_{i j}$, but depend only on a choice of square roots in the construction of a specific cocycle $c$; see (3.12).

Our analysis involves two separate steps:

(I) The spaces spec $A$ and $\operatorname{prim} A$ are homeomorphic to certain sets of ideals of $R$, denoted $\mathcal{G}$-spec $R$ and $\mathcal{G}$-max $R$, which are equipped with Zariski topologies.

(II) We obtain topological quotient maps $\operatorname{spec} R \rightarrow \mathcal{G}$-spec $R$ and $\max R \rightarrow$ $\mathcal{G}$-max $R$ by sending each prime (respectively, maximal) ideal $P$ of $R$ to the largest member of $\mathcal{G}$-spec $R$ contained within $P$.

Step II works very generally, and so we begin with that. Section 1 contains an axiomatic treatment that produces topological quotients of prime and primitive spectra in a noetherian ring $R$, using certain families of ideals of $R$. (When we say that a ring is noetherian, we mean left and right noetherian.) In Section 2, we restrict to the case of a commutative polynomial ring $R$ over $k$ and a family of ideals stable under a suitably compatible family of groups of automorphisms of $R$. This last setup yields the topological quotients of $\operatorname{spec} R$ and $\max R$ that will appear in our main theorem. Step I is developed in Sections 3 and 4 . Section 3 is devoted to the case of quantum tori, which yields the necessary information about individual "strata" of prime and primitive ideals in $A$; in Section 4 , we glue these results together over the whole prime and primitive spectra of $A$, and obtain the main theorem. Section 5 contains explicit calculations of the resulting topological quotient maps $k^{n} \rightarrow \operatorname{prim} A$ for the case where $A$ is a single parameter quantum affine space, and Section 6 applies the main theorem to twists of more general graded commutative algebras. 
We remind the reader that a map $\phi: X \rightarrow Y$ between topological spaces is a topological quotient map provided $\phi$ is surjective and the topology on $Y$ coincides with the quotient topology induced by $\phi$, that is, a subset $W \subseteq Y$ is closed in $Y$ precisely when $\phi^{-1}(W)$ is closed in $X$. When such a map exists, $Y$ is completely determined (up to homeomorphism) by $X$ and the fibers of $\phi$.

\section{Quotients OF PRIME SPECTRA}

We begin the work of this paper by specifying sufficient conditions under which a collection of ideals in a noetherian ring $R$, equipped with a Zariski topology, will form a topological quotient of the prime spectrum of $R$. These conditions are modelled on standard properties of the collection of those ideals of $R$ which are prime relative to the ideals invariant under some set of operators, such as the collection of $G$-prime ideals where $G$ is a group of automorphisms of $R$, or the collection of $\Delta$-prime ideals where $\Delta$ is a set of derivations on $R$. Conditions for a subcollection to be a topological quotient of the primitive or the maximal spectrum of $R$ are also given. At the end of the section, we specialize to the case of $G$-prime ideals, this being the case we shall need for application to quantum tori.

1.1. Let $R$ be a noetherian ring, and let $X$-spec $R$ be a nonempty set of ideals of $R$. The elements of $X$-spec $R$ will be referred to as $X$-prime ideals, and intersections of collections of $X$-prime ideals will be termed $X$-semiprime ideals. We will further assume, throughout this section, that

(a) For each prime ideal $P$ of $R$ there exists a (unique) $X$-prime ideal, denoted $(P: X)$, that is maximum among $X$-semiprime ideals contained in $P$. In other words, $(P: X)$ is an $X$-prime ideal contained in $P$ and containing all $X$-semiprime ideals which are contained in $P$.

(b) If $P$ is a prime ideal of $R$ minimal over an $X$-prime ideal $Q$, then $Q=(P: X)$. In particular, for each $X$-prime ideal $Q$ there exists a prime ideal $P$ such that $Q=(P: X)$.

1.2. If $I$ is an ideal of $R$ then $V(I)$ will denote the set of prime ideals containing $I$. The set of prime ideals of $R$ will be denoted spec $R$ and will be equipped with the standard Zariski topology: The closed subsets are precisely those of the form $V(I)$, for ideals $I$ of $R$. The sets of (left) primitive and maximal ideals of $R$ will be denoted prim $R$ and $\max R$ respectively, and each of these sets will be given the relative topology from $\operatorname{spec} R$.

1.3 Lemma. Let $Q$ be an $X$-prime ideal in $R$, and let $I$ and $J$ be $X$-semiprime ideals of $R$ such that $I J \subseteq Q$. Then $I$ or $J$ is contained in $Q$.

Proof. Choose a prime ideal $P$ such that $Q=(P: X)$. Then $I J \subseteq P$, and so one of $I$ or $J$ is contained in $P$, say $I \subseteq P$. But $Q$ is the unique maximum $X$-semiprime ideal contained in $P$, and so $I \subseteq Q$.

1.4. If $I$ is an ideal of $R$, define $V_{X}(I)$ to be the set of $X$-prime ideals of $R$ containing $I$; note that $V_{X}(I)=V_{X}(J)$, where $J$ is the intersection of the $X$-prime ideals of $R$ containing $I$ (with the convention that the intersection of an empty collection of ideals is equal to $R$ itself). By (1.3), if $J_{1}, \ldots, J_{t}$ are $X$-semiprime ideals of $R$, then $V_{X}\left(J_{1}\right) \cup \cdots \cup V_{X}\left(J_{t}\right)=V_{X}\left(J_{1} \cap \cdots \cap J_{t}\right)$. Therefore, the standard Zariski topology generalizes to a topology on $X$-spec $R$ : The closed sets are those subsets of the form $V_{X}(I)$, for ideals $I$ of $R$. 
1.5. By our assumptions in (1.1), there is a surjection

$$
\pi: \operatorname{spec} R \rightarrow X-\operatorname{spec} R
$$

sending each prime ideal $P$ to $(P: X)$. We first record two trivial observations concerning the behavior of $\pi$ with respect to closed sets.

Lemma. Let $J$ be an $X$-semiprime ideal of $R$.

(a) $\pi(V(J))=V_{X}(J)$.

(b) $\pi^{-1}\left(V_{X}(J)\right)=V(J)$.

1.6. A subset $U$ of $\operatorname{spec} R$ will be termed $\pi$-stable provided $U$ is a union of fibers of $\pi$, or equivalently, provided $U=\pi^{-1}(\pi(U))$.

Lemma. Let $U$ be a closed $\pi$-stable subset of $\operatorname{spec} R$. Then there exists an $X$ semiprime ideal $J$ of $R$ such that $U=V(J)$.

Proof. Choose an ideal $I$ of $R$ such that $U=V(I)$, and let $P_{1}, \ldots, P_{t}$ be the prime ideals minimal over $I$. Then $P_{1}, \ldots, P_{t}$ are the minimal elements of $U$. For $1 \leq i \leq t$, set $Q_{i}=\left(P_{i}: X\right)$, and set $J=Q_{1} \cap \cdots \cap Q_{t}$. Observe that $U \subseteq V(J)$. Next, let $P$ be a prime ideal minimal over $J$. Note that $P$ is minimal over $Q_{i}$, for some $1 \leq i \leq t$, and so $(P: X)=Q_{i}=\left(P_{i}: X\right)$. Therefore, since $U$ is $\pi$-stable, $P \in U$. Consequently, $V(J) \subseteq U$, and hence $U=V(J)$.

1.7 Proposition. (a) $\pi$ is continuous.

(b) $\pi$ maps $\pi$-stable closed subsets of $\operatorname{spec} R$ to closed subsets of $X$-spec $R$.

(c) $\pi$ is a topological quotient map.

Proof. (a) Let $V$ be a closed subset of $X$-spec $R$. As seen in (1.4), there exists an $X$-semiprime ideal $J$ in $R$ such that $V=V_{X}(J)$. By $(1.5 \mathrm{~b}), \pi^{-1}(V)=V(J)$.

(b) Let $U$ be a $\pi$-stable closed subset of spec $R$. It follows from (1.6) that there exists an $X$-semiprime ideal $J$ in $R$ such that $U=V(J)$. By (1.5a), $\pi(U)=V_{X}(J)$.

(c) Assume that $V$ is a subset of $X$-spec $R$ whose inverse image $U=\pi^{-1}(V)$ is closed in $\operatorname{spec} R$. It only remains to show that $V$ is closed. However, $U$ is clearly $\pi$-stable, and so $V$ is closed by (b).

1.8. Let us say that a subset $U$ of $\operatorname{prim} R$ (or of $\max R$ ) is relatively $\pi$-stable provided $U=V \cap \operatorname{prim} R$ (or $U=V \cap \max R$ ) for some $\pi$-stable subset $V$ of $\operatorname{spec} R$.

Proposition. (a) Suppose that $\cap U$ is $X$-semiprime for every relatively $\pi$-stable subset $U$ of $\operatorname{prim} R$. Then the restriction of $\pi$ to the surjection

$$
\operatorname{prim} R \longrightarrow \pi(\operatorname{prim} R)
$$

is a topological quotient map (with respect to the relative topologies).

(b) Similarly, if the intersection of any relatively $\pi$-stable subset of $\max R$ is $X$-semiprime, then $\pi$ restricts to a topological quotient map

$$
\max R \longrightarrow \pi(\max R) \text {. }
$$

Proof. (a) Let $\pi_{\text {prim }}: \operatorname{prim} R \rightarrow \pi(\operatorname{prim} R)$ denote the restriction of $\pi$. It is immediate from the continuity of $\pi$ that $\pi_{\text {prim }}$ is continuous. Now let $W$ be a subset of $\pi(\operatorname{prim} R)$ whose inverse image $U=\pi_{\text {prim }}^{-1}(W)$ is closed in $\operatorname{prim} R$. Since $U$ is relatively $\pi$-stable, its intersection is by hypothesis an $X$-semiprime ideal $J$. Moreover, $U=V(J) \cap \operatorname{prim} R$ because $U$ is closed in $\operatorname{prim} R$. Then $W=\pi(U) \subseteq \pi(V(J))=V_{X}(J)$. If $I \in V_{X}(J) \cap \pi(\operatorname{prim} R)$, there exists $P \in \operatorname{prim} R$ 
such that $\pi(P)=I \supseteq J$. Since $P \supseteq \pi(P)$, we have $P \in V(J) \cap \operatorname{prim} R=U$, and so $I \in W$. Thus $W=V_{X}(J) \cap \pi(\operatorname{prim} R)$, a relatively closed subset of $\pi(\operatorname{prim} R)$.

(b) Use the proof above, with "prim" replaced by "max" everywhere.

The preceding observations apply easily to group actions, as follows.

1.9. For the remainder of this section, let $G$ be a group acting by automorphisms on $R$. An ideal $I$ of $R$, such that $g(I)=I$ for all $g \in G$, is termed a $G$-ideal. Note that the set of $G$-ideals of $R$ is closed under products, sums, and intersections. A (set-theoretically) proper $G$-ideal $Q$ of $R$ is $G$-prime provided $I J \nsubseteq Q$ for all $G$ ideals $I$ and $J$ not contained in $Q$. An intersection of $G$-prime ideals will be termed $G$-semiprime. For any ideal $I$ of $R$, set $(I: G)=\bigcap_{g \in G} g(I)$. It is easy to see that $(I: G)$ is the unique maximum $G$-ideal contained in $I$.

1.10 Lemma (cf. [5, Remarks $\left.4^{*}, 5^{*}\right]$ ). (a). Let $P$ be a prime ideal of $R$. Then $(P: G)$ is a $G$-prime ideal.

(b). Let $Q$ be a $G$-prime ideal of $R$, and let $P$ be a prime ideal of $R$ minimal over $Q$. Then $Q=(P: G)$, and every prime ideal minimal over $Q$ is in the $G$-orbit of $P$. In particular, $Q$ is semiprime.

Proof. Part (a) follows from an argument mimicking the proof of (1.3).

(b) Let $P_{1}, \ldots, P_{t}$ be the prime ideals of $R$ minimal over $Q$, and set $N_{i}=\left(P_{i}: G\right)$ for each $1 \leq i \leq t$. Note that $N_{1}, \ldots, N_{t}$ are $G$-ideals, all containing $Q$, and that some product of them is contained within $Q$. Therefore, $Q=N_{i}$ for some $i$, because $Q$ is $G$-prime. Now consider an arbitrary prime ideal $P_{j}$ minimal over $Q$. Since $P_{j} \supseteq Q=N_{i}$, we see that $P_{j}$ contains a product of prime ideals in the $G$-orbit of $P_{i}$, and so $P_{j} \supseteq g\left(P_{i}\right) \supseteq Q$ for some $g \in G$. Therefore, $P_{j}=g\left(P_{i}\right)$, and $Q=\left(P_{i}: G\right)=\left(P_{j}: G\right)$. Part (b) follows.

1.11. Let $G$-spec $R$ denote the set of $G$-prime ideals of $R$. It follows from (1.9) and (1.10) that $G$-spec $R$ satisfies the axioms for $X$-spec $R$ specified in (1.1). In particular, if $V_{G}(I)$ denotes the set of $G$-prime ideals of $R$ that contain a given ideal $I$ of $R$, then by (1.4) there is a Zariski topology on $G$-spec $R$ : The closed sets are those of the form $V_{G}(I)$, for ideals $I$ of $R$. With respect to this topology, we can use $(1.7 \mathrm{c})$ to deduce that the assignment

$$
P \mapsto(P: G)
$$

is a topological quotient map from $\operatorname{spec} R$ onto $G$-spec $R$.

Let $G$-max $R$ denote the set of maximal proper $G$-ideals. Observe that $G$-max $R$ is a subset of $G$-spec $R$ and that $G$-max $R$ is comprised of the maximal members of $G$-spec $R$. Equip $G$-max $R$ with the relative topology. While each member of $G$-max $R$ must be equal to $(M: G)$ for some $M \in \max R$, the converse fails in general. For example, let $R$ be a polynomial ring $k[x]$ over an infinite field $k$, and let $G=k^{\times}$act on $R$ by the rule $g \cdot f(x)=f(g x)$. Then $(\langle x-\alpha\rangle: G)=0$ for all nonzero $\alpha \in k$, whereas $G$-max $R$ consists of the single maximal ideal $\langle x\rangle$.

In the present setting, the hypothesis of $(1.8 \mathrm{~b})$ is easily verified, as follows. Suppose $U$ is a relatively $\pi$-stable subset of $\max R$, and set $J=\bigcap U$. Since $\max R$ is stable under the action of $G$ (within $\operatorname{spec} R$ ), so is $U$, and hence $J=\bigcap_{M \in U}(M: G)$. Thus by (1.10a), $J$ is $G$-semiprime, as desired. We therefore conclude from (1.8b) that the assignment

$$
M \mapsto(M: G)
$$


is a topological quotient map from $\max R$ onto $\pi(\max R)$.

1.12. Suppose that $G$ has no proper subgroups of finite index. Then all finite $G$ orbits of ideals of $R$ are singletons, and so all $G$-prime ideals of $R$ are prime, by (1.10b). We see, in this case, that $G$-spec $R$ is a subset of $\operatorname{spec} R$ and that the topology on $G$-spec $R$ described in (1.11) is the relative topology.

1.13. We conclude this section by considering a particular case of the group action setting that will be needed later.

Let $k$ be an algebraically closed field, $R=k\left[y_{1}^{ \pm 1}, \ldots, y_{n}^{ \pm 1}\right]$ a Laurent polynomial ring over $k$, and $H=\left(k^{\times}\right)^{n}$ the standard algebraic $k$-torus of rank $n$. Let $H$ act on $R$ by $k$-algebra automorphisms in the natural manner; namely,

$$
\left(h_{1}, \ldots, h_{n}\right) \cdot f\left(y_{1}, \ldots, y_{n}\right)=f\left(h_{1} y_{1}, \ldots, h_{n} y_{n}\right)
$$

for $\left(h_{1}, \ldots, h_{n}\right) \in H$ and $f\left(y_{1}, \ldots, y_{n}\right) \in R$. Since $k$ is algebraically closed, the induced action of $H$ on $\max R$ is transitive.

Proposition. Let $G$ be a subgroup of $H$.

(a) The set $\{(M: G) \mid M \in \max R\}$ coincides with $G$-max $R$.

(b) If $G$ is a closed subgroup of $H$, the fibers of the map $M \mapsto(M: G)$, from $\max R$ to $G-\max R$, are precisely the $G$-orbits in $\max R$.

Proof. (a) Given $Q \in G-\max R$, choose a maximal ideal $N \supseteq Q$. Then $(N: G) \supseteq$ $Q$, and so $Q=(N: G)$ by the maximality of $Q$.

Conversely, consider $Q=(M: G)$, where $M \in \max R$. Choose $Q^{\prime} \in G-\max R$ containing $Q$. By the preceding paragraph, there exists a maximal ideal $M^{\prime}$ in $\max R$ for which $Q^{\prime}=\left(M^{\prime}: G\right)$. Since $H$ acts transitively on $\max R$, there exists an $h \in H$ such that $M=h\left(M^{\prime}\right)$. Since $H$ is abelian,

$$
Q=(M: G)=\left(h\left(M^{\prime}\right): G\right)=h\left(Q^{\prime}\right) .
$$

Hence $Q^{\prime} \supseteq h\left(Q^{\prime}\right)$, and so $Q^{\prime}=h\left(Q^{\prime}\right)=Q$. Thus $Q \in G$-max $R$.

(b) Let $M, M^{\prime} \in \max R$. If $M$ and $M^{\prime}$ are in the same $G$-orbit, then $(M: G)=$ $\left(M^{\prime}: G\right)$. Conversely, assume that $(M: G)=\left(M^{\prime}: G\right)$. On intersecting with the ring of $G$-invariants $R^{G}$, we thus obtain

$$
M \cap R^{G}=(M: G) \cap R^{G}=\left(M^{\prime}: G\right) \cap R^{G}=M^{\prime} \cap R^{G} .
$$

Next, we may view $R$ as the coordinate algebra of $H$ and identify $H$ with $\max R$. By [14 Theorem 48, p. 220], for example, there is an identification of $H / G$ with $\max R^{G}$ that produces the following commutative diagram:

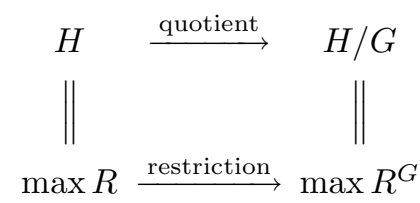

Letting $H$ act on itself and on $H / G$ by right translation (i.e., $g \in H$ acts on $h \in H$ by $g . h=h g^{-1}$ ), the diagram above is $H$-equivariant.

Since the cosets of $G$ in $H$ are the $G$-orbits with respect to right translation, it follows that the fibers of the restriction map $\max R \rightarrow \max R^{G}$ are the $G$-orbits in $\max R$. Therefore $M$ and $M^{\prime}$ lie in the same $G$-orbit, as desired. 


\section{Quotients of AFFine SPACE}

Throughout this section, $k$ denotes a field, and $R$ denotes a commutative polynomial ring $k\left[y_{1}, \ldots, y_{n}\right]$. We study a "piecewise" action on $R$ by a compatible system of groups, together with a collection of ideals which we show satisfies the axioms of the previous section. The resulting quotients of the prime and maximal spectra of $R$ will play a crucial role in our main theorem. Namely, as we shall prove in Section 4, the quotients of $\operatorname{spec} R$ and $\max R$ with respect to a suitable system of groups turn out to be homeomorphic to the prime and primitive spectra, respectively, of a quantum affine $n$-space.

The setup we develop here amounts to patching together finitely many quotients by group actions, as follows. We first partition $\operatorname{spec} R$ into the $2^{n}$ locally closed sets determined by which subsets of $\left\{y_{1}, \ldots, y_{n}\right\}$ are contained in given prime ideals. These subsets are homeomorphic, via localization, to the prime spectra of the Laurent polynomial rings obtained by factoring some of the $y_{i}$ out of $R$ and inverting the remainder. Our "piecewise group action", finally, amounts to compatible choices of groups acting as automorphisms on the above localizations.

2.1. Set $H=\left(k^{\times}\right)^{n}$, the algebraic $k$-torus of rank $n$, and equip $H$ with the Zariski topology. As in (1.13), let $H$ act on $R$ by the automorphisms

$$
\left(h_{1}, \ldots, h_{n}\right) \cdot f\left(y_{1}, \ldots, y_{n}\right)=f\left(h_{1} y_{1}, \ldots, h_{n} y_{n}\right)
$$

We will also consider the induced actions of $H$ on $\operatorname{spec} R$ and $\max R$. Next, let $W$ denote the set of subsets of $\{1, \ldots, n\}$. For each $w \in W$, let $\operatorname{spec}_{w} R$, equipped with the relative topology, denote the set of prime ideals $P$ of $R$ such that

$$
P \cap\left\{y_{1}, \ldots, y_{n}\right\}=\left\{y_{i} \mid i \in w\right\} .
$$

Note that $\operatorname{spec} R=\bigsqcup_{w \in W} \operatorname{spec}_{w} R$. Also note that $\operatorname{spec}_{w} R$ is stable under $H$, and that there is an obvious $H$-equivariant homeomorphism

$$
\operatorname{spec}_{w} R \stackrel{\cong}{\longrightarrow} \operatorname{spec} k\left[y_{i}^{ \pm 1} \mid i \notin w\right] .
$$

2.2. Let $\mathcal{G}=\left(G_{w}\right)_{w \in W}$ be a family of subgroups of $H$, indexed by $W$, subject to the following compatibility hypothesis: $\left(P: G_{v}\right) \subseteq\left(P: G_{w}\right)$ for all $v \subseteq w$ and every $P \in \operatorname{spec}_{w} R$. We do not exclude the possibility that the $G_{w}$, for $w \in W$, are identically the same. Set

$$
\mathcal{G}-\operatorname{spec}_{w} R=\left\{\left(P: G_{w}\right) \mid P \in \operatorname{spec}_{w} R\right\},
$$

for $w \in W$. Note, since the $y_{i}$ are $G_{w}$-eigenvectors, that $Q \cap\left\{y_{1}, \ldots, y_{n}\right\}=$ $\left\{y_{i} \mid i \in w\right\}$ for all $Q \in \mathcal{G}-\operatorname{spec}_{w} R$. Consequently, the sets $\mathcal{G}-\operatorname{spec}_{w} R$ for $w \in W$ are pairwise disjoint, and we set

$$
\mathcal{G}-\operatorname{spec} R=\bigsqcup_{w \in W} \mathcal{G}-\operatorname{spec}_{w} R .
$$

The members of $\mathcal{G}$-spec $R$ will be termed $\mathcal{G}$-prime ideals, the intersections of collections of $\mathcal{G}$-prime ideals will be called $\mathcal{G}$-semiprime ideals, and the set of $\mathcal{G}$-prime ideals containing an ideal $I$ of $R$ will be denoted $V_{\mathcal{G}}(I)$. Note from $(1.10 \mathrm{~b})$ that all $\mathcal{G}$-semiprime ideals are semiprime.

2.3 Lemma. $\mathcal{G}$-spec $R$ satisfies the conditions in $(1.1)$, with $(P: \mathcal{G})=\left(P: G_{w}\right)$ for $P \in \operatorname{spec}_{w} R$. 
Proof. To verify (1.1a), let $P \in \operatorname{spec}_{w} R$, let $Q=\left(P: G_{w}\right)$, and let $Q^{\prime}$ be an arbitrary $\mathcal{G}$-semiprime ideal of $R$ contained in $P$. We will show that $Q^{\prime} \subseteq Q$. To start, write

$$
Q^{\prime}=\bigcap_{v \in W} N_{v}
$$

where each $N_{v}$ is an intersection of ideals from $\mathcal{G}-\operatorname{spec}_{v} R$, and where each $N_{v}$ contains $Q^{\prime}$. Since $Q^{\prime} \subseteq P$, some $N_{v} \subseteq P$. Then, since $y_{i} \in N_{v}$ for all $i \in v$, we must have $v \subseteq w$. Note that $N_{v}$ is $G_{v}$-stable and so is contained in $\left(P: G_{v}\right)$. By the compatibility hypothesis, $N_{v} \subseteq Q$, whence $Q^{\prime} \subseteq Q$, and (1.1a) is established.

To check (1.1b), let $Q \in \mathcal{G}-\operatorname{spec}_{w} R$ and let $P$ be a prime ideal of $R$ minimal over $Q$. Observe first that $P \in \operatorname{spec}_{v} R$ for some $v \supseteq w$. Next, it follows from (1.10a) that $Q$ is $G_{w}$-prime, and then from (1.10b) that $Q=\left(P: G_{w}\right)$. Moreover, since each of the variables $y_{i}$ is a $G_{w}$-eigenvector, it now follows that $v=w$, whence $Q=(P: \mathcal{G})$ and $(1.1 \mathrm{~b})$ is satisfied.

2.4. Following (1.4), we equip $\mathcal{G}$-spec $R$ with the Zariski topology where the closed subsets are the $V_{\mathcal{G}}(I)$, for ideals $I$ of $R$. In case none of the $G_{w}$ has proper subgroups of finite index, it follows from (1.12) that $\mathcal{G}$-spec $R \subseteq \operatorname{spec} R$, and then the topology on $\mathcal{G}$-spec $R$ coincides with the relative topology.

Note, for each $w \in W$, that $\operatorname{spec}_{w} R$ is a union of $H$-orbits. Therefore, since $H$ is abelian, we see that $h(P: \mathcal{G})=(h(P): \mathcal{G})$ for all $h \in H$ and $P \in \operatorname{spec} R$. From (1.7) we now obtain:

Proposition. The assignment $P \mapsto(P: \mathcal{G})$ produces an $H$-equivariant topological quotient map from $\operatorname{spec} R$ onto $\mathcal{G}$-spec $R$.

2.5. For $w \in W$, set $\max _{w} R=\max R \cap \operatorname{spec}_{w} R$. Note that

$$
\max R=\bigsqcup_{w \in W} \max _{w} R \text {. }
$$

Moreover, $\max _{w} R$ is equal to the set of maximal members of $\operatorname{spec}_{w} R$. Next, set

$$
\begin{aligned}
\mathcal{G}-\max _{w} R & =\left\{\left(M: G_{w}\right) \mid M \in \max _{w} R\right\} \quad(\text { for } w \in W) ; \\
\mathcal{G}-\max R & =\bigsqcup_{w \in W} \mathcal{G}-\max _{w} R=\{(M: \mathcal{G}) \mid M \in \max R\} .
\end{aligned}
$$

Note that the definition of $\mathcal{G}-\max _{w} R$ above and the definition of $G_{w}$-max $R$ in (1.11) follow different patterns. However, we shall see in (2.6) that when $k$ is algebraically closed, $\mathcal{G}-\max _{w} R$ coincides with $\left(G_{w}-\max R\right) \cap\left(\mathcal{G}-\operatorname{spec}_{w} R\right)$.

Proposition. The assignment $M \mapsto(M: \mathcal{G})$ produces an $H$-equivariant topological quotient map from $\max R$ onto $\mathcal{G}-\max R$.

Proof. By (1.8b), it suffices to show that if $U$ is any relatively $\pi$-stable subset of $\max R$ and $J=\bigcap U$, then $J$ is $\mathcal{G}$-semiprime.

To start, set $U_{w}=U \cap \max _{w} R$ for $w \in W$, and note that $U_{w}$ is stable under $G_{w}$ since $(g(M): \mathcal{G})=(M: \mathcal{G})$ for all $M \in \max _{w} R$ and $g \in G_{w}$. Hence,

$$
\bigcap U_{w}=\bigcap_{M \in U_{w}}\left(M: G_{w}\right)=\bigcap_{M \in U_{w}}(M: \mathcal{G}),
$$

which is $\mathcal{G}$-semiprime. Therefore $J=\bigcap_{w \in W}\left(\bigcap U_{w}\right)$ is a $\mathcal{G}$-semiprime ideal, as required. 
2.6 Proposition. Assume that $k$ is algebraically closed. For $w \in W$, the set $\mathcal{G}-\max _{w} R$ coincides with the set of maximal members of $\mathcal{G}-\operatorname{spec}_{w} R$.

Proof. Set $m=n-|w|$ and $R_{w}=\left(R /\left\langle y_{i} \mid i \in w\right\rangle\right)\left[y_{j}^{-1} \mid j \notin w\right]$. Note that $R_{w}$ is a Laurent polynomial ring in the (images of the) $m$ indeterminates $y_{j}$, for $j \notin w$. We thereby obtain an action of the $m$-torus $\left(k^{\times}\right)^{m}$ on $R_{w}$, following (1.13). Note that the actions on $R_{w}$ by $G_{w}$ and $H$, induced from their actions on $R$, factor through the $\left(k^{\times}\right)^{m}$-action. Moreover, localization provides $H$-equivariant bijections

$$
\max _{w} R \rightarrow \max R_{w} \quad \text { and } \quad \mathcal{G}-\operatorname{spec}_{w} R \rightarrow G_{w}-\operatorname{spec} R_{w} .
$$

The proposition therefore follows from (1.13a).

2.7. We close this section by considering the fibers of the map in (2.5) more closely. First note that each $\max _{w} R$ is a disjoint union of fibers. Next, on $\max _{w} R$ the map is given by the rule $M \mapsto\left(M: G_{w}\right)$, and so the fibers within $\max _{w} R$ are unions of $G_{w}$-orbits. Our interest is in conditions under which the fibers within $\max _{w} R$ coincide with the $G_{w}$-orbits.

The maximal ideals in $\max _{w} R$ have the form

$$
\left\langle y_{i} \mid i \in w\right\rangle+\left\langle f_{1}, \ldots, f_{t}\right\rangle
$$

where $f_{1}, \ldots, f_{t} \in k\left[y_{j} \mid j \notin w\right]$. These ideals are all fixed by the group

$$
H_{w}=\left\{\left(h_{1}, \ldots, h_{n}\right) \in H \mid h_{j}=1 \text { for all } j \notin w\right\} ;
$$

thus $\left(M: G_{w}\right)=\left(M: G_{w} H_{w}\right)$ for all $M \in \max _{w} R$. Hence, there is no loss of generality in assuming that $G_{w} \supseteq H_{w}$.

Proposition. Fix $w \in W$, and assume that $k$ is algebraically closed. Assume that $G_{w} \supseteq H_{w}$ and that $G_{w} / H_{w}$ is a closed subgroup of $H / H_{w}$. Then the fibers of the map $M \mapsto(M: \mathcal{G})$, from $\max _{w} R$ to $\mathcal{G}-\max _{w} R$, are precisely the $G_{w}$-orbits in $\max _{w} R$.

Proof. We shift everything to the localization $R_{w}$, as in the proof of (2.6), and we recall the $\left(k^{\times}\right)^{m}$-action on $R_{w}$ described therein, where $m=n-|w|$. Now observe that the induced action of $H / H_{w}$ on $R_{w}$ is identical to the $\left(k^{\times}\right)^{m}$-action on $R_{w}$. Therefore, the proposition follows from (1.13b).

\section{QUANTUM TORI}

As in [6], much of our analysis of quantum affine spaces can be reduced, via localization, to quantum tori. This portion of our analysis is carried out in the present section. It leads, in particular, to a version of our main theorem for an arbitrary multiparameter quantum torus $\mathcal{O}_{\mathbf{q}}\left(\left(k^{\times}\right)^{n}\right)$ over an algebraically closed field $k$, which establishes that $\operatorname{spec} \mathcal{O}_{\mathbf{q}}\left(\left(k^{\times}\right)^{n}\right)$ and $\operatorname{prim} \mathcal{O}_{\mathbf{q}}\left(\left(k^{\times}\right)^{n}\right)$ can be presented as topological quotients of $\operatorname{spec} \mathcal{O}\left(\left(k^{\times}\right)^{n}\right)$ and $\max \mathcal{O}\left(\left(k^{\times}\right)^{n}\right)$, respectively. No restriction on the parameter matrix $\mathbf{q}$ is needed here; it is only in patching quantum tori together to cover a quantum affine space, as in the following section, that we will need to avoid -1 .

Let $k$ be a field. From (3.7) onward, we will assume that $k$ is algebraically closed.

3.1. Let $\mathbf{q}=\left(q_{i j}\right)$ be a multiplicatively antisymmetric $n \times n$ matrix over $k$; that is, $q_{i i}=1$ and $q_{j i}=q_{i j}^{-1}$, for all $i, j$. Let $A=\mathcal{O}_{\mathbf{q}}\left(\left(k^{\times}\right)^{n}\right)$ be the corresponding multiparameter quantized coordinate ring of the torus $\left(k^{\times}\right)^{n}$, that is, the $k$-algebra generated by elements $x_{1}^{ \pm 1}, \ldots, x_{n}^{ \pm 1}$ subject only to the relations $x_{i} x_{j}=q_{i j} x_{j} x_{i}$ for 
all $i, j$. This algebra is also known as a McConnell-Pettit algebra, after [13]; in the notation of that paper, $A=P(\mathbf{q})$.

Let us first express $A$ in terms of ordered monomials, using standard multi-index notation. Thus $A$ has a $k$-basis of monomials, $x^{\alpha}=x_{1}^{\alpha_{1}} x_{2}^{\alpha_{2}} \cdots x_{n}^{\alpha_{n}}$, for $n$-tuples $\alpha=\left(\alpha_{1}, \ldots, \alpha_{n}\right)$ from the group $\Gamma:=\mathbb{Z}^{n}$. Define $\sigma: \Gamma \times \Gamma \rightarrow k^{\times}$by

$$
\sigma(\alpha, \beta)=\prod_{i, j=1}^{n} q_{i j}^{\alpha_{i} \beta_{j}}
$$

Then $\sigma$ determines the commutation rules in $A$; namely, $x^{\alpha} x^{\beta}=\sigma(\alpha, \beta) x^{\beta} x^{\alpha}$ for $\alpha, \beta \in \Gamma$. Moreover, $\sigma$ is an alternating bicharacter on $\Gamma$, because

$$
\sigma(\alpha, \alpha)=1, \quad \sigma(\beta, \alpha)=\sigma(\alpha, \beta)^{-1}, \quad \sigma\left(\alpha, \beta+\beta^{\prime}\right)=\sigma(\alpha, \beta) \sigma\left(\alpha, \beta^{\prime}\right),
$$

for $\alpha, \beta, \beta^{\prime} \in \Gamma$.

3.2. It is convenient to view $A$ as a twisted group algebra of $\Gamma$. One way to do this is to use the function $d: \Gamma \times \Gamma \rightarrow k^{\times}$such that $x^{\alpha} x^{\beta}=d(\alpha, \beta) x^{\alpha+\beta}$ for all $\alpha, \beta \in \Gamma$. It follows from the associativity of multiplication in $A$ that $d$ must be a 2 -cocycle on $\Gamma$. Thus by writing $A$ in terms of the $k$-basis $\left\{x^{\alpha} \mid \alpha \in \Gamma\right\}$, we have expressed $A$ as a twisted group algebra $k^{d} \Gamma$.

Note that $\sigma(\alpha, \beta)=d(\alpha, \beta) d(\beta, \alpha)^{-1}$ for $\alpha, \beta \in \Gamma$. Conversely, if $c$ is any 2cocycle on $\Gamma$ such that $c(\alpha, \beta) c(\beta, \alpha)^{-1}=\sigma(\alpha, \beta)$ for $\alpha, \beta \in \Gamma$, then $k^{c} \Gamma \cong A$. Namely, $k^{c} \Gamma$ has a basis $\left\{x_{\alpha} \mid \alpha \in \Gamma\right\}$ such that $x_{\alpha} x_{\beta}=c(\alpha, \beta) x_{\alpha+\beta}$ for $\alpha, \beta \in \Gamma$. If $\epsilon_{1}, \ldots, \epsilon_{n}$ denotes the standard basis for $\Gamma$, then $k^{c} \Gamma$ is generated by the elements $x_{\epsilon_{1}}^{ \pm 1}, \ldots, x_{\epsilon_{n}}^{ \pm 1}$, which satisfy the same commutation rules as $x_{1}, \ldots, x_{n}$, namely

$$
x_{\epsilon_{i}} x_{\epsilon_{j}}=c\left(\epsilon_{i}, \epsilon_{j}\right) x_{\epsilon_{i}+\epsilon_{j}}=c\left(\epsilon_{i}, \epsilon_{j}\right) c\left(\epsilon_{j}, \epsilon_{i}\right)^{-1} x_{\epsilon_{j}} x_{\epsilon_{i}}=\sigma\left(\epsilon_{i}, \epsilon_{j}\right) x_{\epsilon_{j}} x_{\epsilon_{i}}=q_{i j} x_{\epsilon_{j}} x_{\epsilon_{i}}
$$

for all $i, j$. Therefore there exists a $k$-algebra isomorphism $k^{c} \Gamma \rightarrow A$ such that $x_{\epsilon_{i}} \mapsto x_{i}$ for all $i$. Note that this isomorphism sends each $x_{\alpha}$ to a scalar multiple of $x^{\alpha}$.

While the cocycle $d$ is useful for some purposes, it has some drawbacks; for example, it is neither symmetric nor antisymmetric in general. We therefore leave the choice of a particular cocycle until later (see (3.5) and (3.12)).

3.3. Let $c$ be any 2-cocycle on $\Gamma$ such that $c(\alpha, \beta) c(\beta, \alpha)^{-1}=\sigma(\alpha, \beta)$ for $\alpha, \beta \in \Gamma$, and identify $A$ with $k^{c} \Gamma$. This allows us to write $A$ in terms of a $k$-basis $\left\{x_{\alpha} \mid \alpha \in \Gamma\right\}$, with multiplication given by

$$
x_{\alpha} x_{\beta}=c(\alpha, \beta) x_{\alpha+\beta}
$$

for $\alpha, \beta \in \Gamma$. We shall also need the ordinary group algebra $k \Gamma$. Let us express this algebra in terms of a $k$-basis $\left\{y_{\alpha} \mid \alpha \in \Gamma\right\}$, with $y_{\alpha} y_{\beta}=y_{\alpha+\beta}$ for $\alpha, \beta \in \Gamma$.

3.4. Set $H=\operatorname{Hom}\left(\Gamma, k^{\times}\right.$), an algebraic torus of rank $n$ (which will henceforth be equipped with the Zariski topology). Write the application of $H$ to $\Gamma$ in terms of a pairing $\langle-,-\rangle: H \times \Gamma \rightarrow k^{\times}$. Define actions of $H$ on both $A$ and $k \Gamma$ via $k$-algebra automorphisms such that

$$
h . x_{\alpha}=\langle h, \alpha\rangle x_{\alpha} \quad \text { and } \quad h . y_{\alpha}=\langle h, \alpha\rangle y_{\alpha},
$$

for $h \in H$ and $\alpha \in \Gamma$. 
3.5. Let $\Phi_{c}: A \rightarrow k \Gamma$ be the $k$-linear isomorphism such that $\Phi_{c}\left(x_{\alpha}\right)=y_{\alpha}$ for $\alpha \in \Gamma$. Observe that $\Phi_{c}$ is $H$-equivariant. The extent to which $\Phi_{c}$ fails to preserve products can be expressed in terms of $c$ : since $x_{\alpha} x_{\beta}=c(\alpha, \beta) x_{\alpha+\beta}$, we have

$$
\Phi_{c}\left(x_{\alpha} x_{\beta}\right)=c(\alpha, \beta) y_{\alpha+\beta}=c(\alpha, \beta) \Phi_{c}\left(x_{\alpha}\right) \Phi_{c}\left(x_{\beta}\right)
$$

for $\alpha, \beta \in \Gamma$.

As in [6] 1.2], set $S=\operatorname{rad}(\sigma)=\{\alpha \in \Gamma \mid \sigma(\alpha,-) \equiv 1\}$, a subgroup of $\Gamma$; then

$$
Z(A)=k\left[x_{\alpha} \mid \alpha \in S\right] .
$$

If we identify the group algebra $k S$ with the $k$-linear span of $\left\{y_{\alpha} \mid \alpha \in S\right\}$ inside $k \Gamma$, then $\Phi_{c}(Z(A))=k S$. In fact:

Lemma. Assume that $c \equiv 1$ on $S \times \Gamma \subseteq \Gamma \times \Gamma$. Then the map $\Phi_{c}$ restricts to a $k$-algebra isomorphism of $Z(A)$ onto $k S$. With respect to this isomorphism, $\Phi_{c}$ itself gives a semilinear isomorphism of $A$ as $Z(A)$-module onto $k \Gamma$ as $k S$-module: $\Phi_{c}(z a)=\Phi_{c}(z) \Phi_{c}(a)$ for all $z \in Z(A)$ and $a \in A$.

Proof. By assumption, $\Phi_{c}\left(x_{\alpha} x_{\beta}\right)=\Phi_{c}\left(x_{\alpha}\right) \Phi_{c}\left(x_{\beta}\right)$ for $\alpha \in S$ and $\beta \in \Gamma$. Consequently, $\Phi_{c}(z a)=\Phi_{c}(z) \Phi_{c}(a)$ for all $z \in Z(A)$ and $a \in A$, and the lemma follows.

We shall need the following fact to show that $S$ equals the intersection of the kernels of some homomorphisms from $H$ (see (3.7)).

3.6 Lemma. The order of the torsion subgroup of $\Gamma / S$ is not divisible by char $k$.

Proof. The torsion subgroup of $\Gamma / S$ is a finite abelian group, hence a finite direct sum of finite cyclic groups, and so the order of this subgroup equals the product of the orders of certain of its elements. Thus it suffices to show that the order of each torsion element of $\Gamma / S$ is not divisible by char $k$.

Let $\alpha+S$ be a torsion element of $\Gamma / S$, and choose a basis $\epsilon_{1}, \ldots, \epsilon_{n}$ for $\Gamma$. For each positive integer $m$, we have

$$
m \alpha \in S \Longleftrightarrow \sigma\left(m \alpha, \gamma_{i}\right)=1 \text { for all } i \quad \Longleftrightarrow \quad \sigma\left(\alpha, \epsilon_{i}\right)^{m}=1 \text { for all } i .
$$

Hence, the order of $\alpha+S$ equals the order of the element $\left(\sigma\left(\alpha, \epsilon_{1}\right), \ldots, \sigma\left(\alpha, \epsilon_{n}\right)\right)$ in $\left(k^{\times}\right)^{n}$, and the latter order is clearly not divisible by char $k$.

3.7. From now until the end of the section, assume that $k$ is algebraically closed.

We use ${ }^{\perp}$ to denote orthogonals of subsets of $H$ and $\Gamma$, relative to the pairing $\langle-,-\rangle$. In particular,

$$
S^{\perp}=\{h \in H \mid\langle h, \alpha\rangle=1 \text { for } \alpha \in S\}=\{h \in H \mid \operatorname{ker} h \supseteq S\},
$$

a closed subgroup of $H$, and

$$
S^{\perp \perp}=\left\{\alpha \in \Gamma \mid\langle h, \alpha\rangle=1 \text { for } h \in S^{\perp}\right\}=\bigcap_{h \in S^{\perp}} \operatorname{ker} h,
$$

a subgroup of $\Gamma$.

Lemma. $S^{\perp \perp}=S$.

Proof. Consider $\alpha \in \Gamma \backslash S$. The order of the coset $\alpha+S$ in $\Gamma / S$ is either infinite, or - if finite - not divisible by char $k$, in view of (3.6). In either case, the subgroup $\mathbb{Z}(\alpha+S) \subseteq \Gamma / S$ supports a nontrivial homomorphism to $k^{\times}$. Since $k^{\times}$is divisible, any such homomorphism extends to a homomorphism $\Gamma / S \rightarrow k^{\times}$. Thus, there 
exists a homomorphism $h: \Gamma \rightarrow k^{\times}$(i.e., $h \in H$ ) such that $S \subseteq \operatorname{ker} h$ and $h(\alpha) \neq 1$, which verifies that $\alpha \notin S^{\perp \perp}$.

3.8 Proposition. (a) $A^{S^{\perp}}=Z(A)$ and $(k \Gamma)^{S^{\perp}}=k S$.

(b) Every $S^{\perp}$-stable ideal of $k \Gamma$ is generated by its contraction to $k S$.

(c) Every ideal of $A$ is generated by its contraction to $Z(A)$.

(d) Assume that $c \equiv 1$ on $S \times \Gamma$. The map $I \mapsto \Phi_{c}(I)$, for ideals $I$ of $A$, provides a product preserving, $H$-equivariant lattice isomorphism from the lattice of ideals of $A$ onto the lattice of $S^{\perp}$-ideals of $k \Gamma$.

Proof. (a) It follows from (3.7) that $A^{S^{\perp}}$ is spanned by the $x_{\alpha}$ for $\alpha \in S$, and that $(k \Gamma)^{S^{\perp}}$ is spanned by the $y_{\alpha}$ for $\alpha \in S$. The second part of (a) is thus clear, and the first follows from (3.5).

(b) Consider an $S^{\perp}$-stable ideal $I$ in $k \Gamma$. Since $S^{\perp}$ acts semisimply on $k \Gamma$, this ideal must be spanned by $S^{\perp}$-eigenvectors. Let $a \in I$ be an $S^{\perp}$-eigenvector, and choose an element $\gamma$ from the support of $a$. Then $x_{\gamma}^{-1} a$ is also an $S^{\perp}$-eigenvector and contains 1 in its support. Hence $S^{\perp}$ fixes $x_{\gamma}^{-1} a$. Thus $x_{\gamma}^{-1} a \in(k \Gamma)^{S^{\perp}}=k S$, and so $a \in(k \Gamma)(I \cap k S)$. Therefore $I=(k \Gamma)(I \cap k S)$.

(c) See, for example, [6, 1.4].

(d) To start, note that $U \mapsto \Phi_{c}(U)$ produces an $H$-equivariant, bijective lattice isomorphism from the lattice of $k$-subspaces $U$ of $A$ onto the lattice of $k$-subspaces of $k \Gamma$.

Next, let $I$ be an ideal of $A$. It follows from (c) that $I=I^{\prime} A$ for some ideal $I^{\prime}$ of $Z(A)$. By (3.5), $\Phi_{c}\left(I^{\prime}\right)$ is an ideal of $k S$, and $\Phi_{c}(I)=\Phi_{c}\left(I^{\prime}\right) \Phi_{c}(A)=\Phi_{c}\left(I^{\prime}\right) k \Gamma$ is an ideal of $k \Gamma$. Furthermore, since $\Phi_{c}\left(I^{\prime}\right)$ is an ideal of $k S$, and since $k S$ is fixed pointwise by $S^{\perp}$, we see that $\Phi_{c}\left(I^{\prime}\right) k \Gamma$ is stable under $S^{\perp}$. Similarly, it follows from (b) and (3.5) that $\Phi_{c}^{-1}$ maps each $S^{\perp}$-ideal of $k \Gamma$ to an ideal of $A$. Therefore the set maps $\Phi_{c}$ and $\Phi_{c}^{-1}$ provide mutually inverse bijections between the lattices of ideals of $A$ and $S^{\perp}$-ideals of $k \Gamma$. It follows easily from the preceding paragraph that these bijections are $H$-equivariant lattice isomorphisms.

Now let $I$ and $J$ be ideals of $A$. It only remains to show that $\Phi_{c}(I J)=$ $\Phi_{c}(I) \Phi_{c}(J)$. As above, write $I=I^{\prime} A$ and $J=J^{\prime} A$, for ideals $I^{\prime}$ and $J^{\prime}$ of $Z(A)$. Then $I J=I^{\prime} J^{\prime} A$, and so by $(3.5), \Phi_{c}(I J)=\Phi_{c}\left(I^{\prime}\right) \Phi_{c}\left(J^{\prime}\right) k \Gamma=\Phi_{c}(I) \Phi_{c}(J)$.

3.9. Let $S^{\perp}$-spec $k \Gamma$ and $S^{\perp}$ - max $k \Gamma$ denote the sets of $S^{\perp}$-prime and maximal proper $S^{\perp}$-ideals of $k \Gamma$, respectively. As noted in (1.11), $S^{\perp}-\max k \Gamma \subseteq S^{\perp}$ - spec $k \Gamma$. Equip these sets with their respective Zariski topologies, again following (1.11).

Proposition. Assume that $c \equiv 1$ on $S \times \Gamma$. The set maps $\Phi_{c}$ and $\Phi_{c}^{-1}$ provide pairs of mutually inverse, $H$-equivariant, homeomorphisms

$$
\operatorname{spec} A \longleftrightarrow S^{\perp}-\operatorname{spec} k \Gamma \quad \text { and } \quad \max A \longleftrightarrow S^{\perp}-\max k \Gamma \text {. }
$$

Proof. This follows from $(3.8 \mathrm{~d})$.

3.10 Proposition. The assignment $P \mapsto\left(P: S^{\perp}\right)$, for prime ideals $P$ of $k \Gamma$, produces $H$-equivariant topological quotient maps

$$
\operatorname{spec} k \Gamma \rightarrow S^{\perp}-\operatorname{spec} k \Gamma \quad \text { and } \quad \max k \Gamma \rightarrow S^{\perp}-\max k \Gamma \text {. }
$$

Proof. By (1.13a), $S^{\perp-\max } k \Gamma=\left\{\left(M: S^{\perp}\right) \mid M \in \max k \Gamma\right\}$, and so the given assignment does map $\max k \Gamma$ onto $S^{\perp}-\max k \Gamma$. The given maps are $H$-equivariant because $H$ is abelian. The remainder of the proposition follows from (1.11). 
The two propositions above provide the information necessary to exhibit $\operatorname{spec} A$ and $\operatorname{prim} A$ as topological quotients of their classical analogs, as in the following theorem. A cocycle $c$ with the required condition is constructed in (3.12).

3.11 Theorem. Assume that $c \equiv 1$ on $S \times \Gamma$. Then the rule $P \mapsto \Phi_{c}^{-1}\left(P: S^{\perp}\right)$, for prime ideals $P$ of $k \Gamma$, defines $H$-equivariant topological quotient maps

$$
\operatorname{spec} k \Gamma \rightarrow \operatorname{spec} A \quad \text { and } \quad \max k \Gamma \rightarrow \max A .
$$

The fibers of the second map are exactly the $S^{\perp}$-orbits in $\max k \Gamma$.

Proof. The first part follows from (3.9) and (3.10). The final part is a consequence of (1.13b).

3.12. In order to apply (3.11), we must be able to choose the cocycle $c$ to be trivial on $S \times \Gamma$. This can be done as follows:

Lemma. There exists a 2-cocycle $c$ on $\Gamma$ such that $c \equiv 1$ on $S \times \Gamma$ and such that $c(\alpha, \beta) c(\beta, \alpha)^{-1}=\sigma(\alpha, \beta)$ for $\alpha, \beta \in \Gamma$.

Proof. As in [6, 1.6], $\Gamma$ has a basis $\gamma_{1}, \ldots, \gamma_{n}$ such that $S$ is generated by $m_{1} \gamma_{1}, \ldots$, $m_{t} \gamma_{t}$, for some $t \leq n$ and some positive integers $m_{i}$. Set $p_{i j}=\sigma\left(\gamma_{i}, \gamma_{j}\right)$ for all $i, j$, and note that the matrix $\left(p_{i j}\right)$ is multiplicatively antisymmetric. Given $\alpha, \beta \in \Gamma$, write $\alpha=\sum_{i} a_{i} \gamma_{i}$ and $\beta=\sum_{j} b_{j} \gamma_{j}$ for some $a_{i}, b_{j} \in \mathbb{Z}$; then

$$
\sigma(\alpha, \beta)=\prod_{i, j} p_{i j}^{a_{i} b_{j}}
$$

Now define

$$
c(\alpha, \beta)=\prod_{i<j} p_{i j}^{a_{i} b_{j}}
$$

for $\alpha=\sum_{i} a_{i} \gamma_{i}$ and $\beta=\sum_{j} b_{j} \gamma_{j}$ in $\Gamma$. Then $c$ is a bicharacter on $\Gamma$, and hence a 2 -cocycle. It is easily checked that $c(\alpha, \beta) c(\beta, \alpha)^{-1}=\sigma(\alpha, \beta)$ for $\alpha, \beta \in \Gamma$. Since $m_{i} \gamma_{i} \in S$, we obtain

$$
1=\sigma\left(m_{i} \gamma_{i}, \gamma_{j}\right)=p_{i j}^{m_{i}}
$$

for all $i, j$. Hence, if $\alpha=\sum_{i} a_{i} \gamma_{i} \in S$, then $p_{i j}^{a_{i}}=1$ for all $i, j$, and therefore $c(\alpha, \beta)=1$ for all $\beta \in \Gamma$.

3.13 Remark. It would result in a stronger theorem if we could conclude in (3.11) that the given maps were closed. However, this conclusion is not possible in general. For instance, take $n=3$, choose $p \in k^{\times}$not a root of unity, and take

$$
\mathbf{q}=\left(\begin{array}{ccc}
1 & 1 & 1 \\
1 & 1 & p^{2} \\
1 & p^{-2} & 1
\end{array}\right)
$$

Then $\sigma$ is given by the rule $\sigma(s, t)=p^{2\left(s_{2} t_{3}-s_{3} t_{2}\right)}$, and we may choose $c$ to be given by

$$
c(s, t)=p^{s_{2} t_{3}-s_{3} t_{2}} .
$$

We compute that $S=\mathbb{Z}(1,0,0)$ and $S^{\perp}=\left\{\left(h_{1}, h_{2}, h_{3}\right) \in H \mid h_{1}=1\right\}$.

Now write $k \Gamma=k\left[y_{1}^{ \pm 1}, y_{2}^{ \pm 1}, y_{3}^{ \pm 1}\right]$, where $y_{1}, y_{2}, y_{3}$ correspond to the standard basis for $\Gamma$, and let

$$
V=\left\{\left\langle y_{1}-\alpha_{1}, y_{2}-\alpha_{2}, y_{3}-\alpha_{3}\right\rangle \mid \alpha_{i} \in k^{\times} \text {and }\left(\alpha_{1}-1\right) \alpha_{2}=1\right\},
$$


a closed subset of $\max k \Gamma$. The map $M \mapsto\left(M: S^{\perp}\right)$ sends $V$ to the set

$$
\left\{\left\langle y_{1}-\alpha_{1}\right\rangle \mid \alpha_{1} \in k^{\times} \text {and } \alpha_{1} \neq 1\right\} \text {, }
$$

a non-closed subset of $S^{\perp}-\max k \Gamma$, and thus $M \mapsto \Phi_{c}^{-1}\left(M: S^{\perp}\right)$ sends $V$ to a non-closed subset of $\max A$.

\section{QuAntum AFFine SPACES}

We now present and prove the main results of this paper. In essence, we partition the prime and primitive spectra of a quantum affine space into corresponding spectra of quantum tori, apply the results of the previous section to these spectra, and then patch everything together. It is the patching process that requires a more careful choice of cocycle than previously.

We continue to assume that the base field $k$ is algebraically closed.

4.1. Again let $\mathbf{q}=\left(q_{i j}\right)$ be a multiplicatively antisymmetric $n \times n$ matrix over $k$. Now, however, let $A=\mathcal{O}_{\mathbf{q}}\left(k^{n}\right)$ be the corresponding multiparameter quantized coordinate ring of affine $n$-space. In other words, $A$ is the $k$-algebra generated by elements $x_{1}, \ldots, x_{n}$, subject only to the relations $x_{i} x_{j}=q_{i j} x_{j} x_{i}$. We will treat $A$ as a twisted semigroup algebra, as follows.

Set $\Gamma=\mathbb{Z}^{n}$ and $\Gamma^{+}=\left(\mathbb{Z}^{+}\right)^{n}$, and define an alternating bicharacter $\sigma$ on $\Gamma$ by the rule

$$
\sigma(\alpha, \beta)=\prod_{i, j=1}^{n} q_{i j}^{\alpha_{i} \beta_{j}}
$$

as in (3.1). If $c$ is any 2-cocycle on $\Gamma$ such that $c(\alpha, \beta) c(\beta, \alpha)^{-1}=\sigma(\alpha, \beta)$ for $\alpha, \beta \in \Gamma$, we can write $A$ as the twisted semigroup algebra $k^{c} \Gamma^{+}$, viewed in the obvious way as a subalgebra of the twisted group algebra $k^{c} \Gamma$.

We will need to apply the results of Section 3 to the localizations

$$
A_{w}=\left(A /\left\langle x_{i} \mid i \in w\right\rangle\right)\left[x_{j}^{-1} \mid j \notin w\right]
$$

as $w$ ranges over the subsets of $\{1, \ldots, n\}$. Thus, we require a cocycle $c$ whose restrictions to the subgroups of $\Gamma$ generated by subsets of the standard basis all satisfy the hypothesis of (3.11). The cocycle obtained in (3.12) is not sufficient for this purpose. With a minor technical assumption, we can construct a suitable cocycle as follows.

4.2. Let $\left\langle q_{i j}\right\rangle$ denote the subgroup of $k^{\times}$generated by the $q_{i j}$. Assume from now on that either $-1 \notin\left\langle q_{i j}\right\rangle$ or $\operatorname{char} k=2$.

Lemma. There exists an alternating bicharacter $c$ on $\Gamma$ such that $c^{2}=\sigma$ and such that $c(\alpha, \beta)=1$ whenever $\sigma(\alpha, \beta)=1$.

Proof. Since $k$ is algebraically closed, the abelian group $k^{\times}$is divisible, and so it contains a divisible hull $D$ for the subgroup $\left\langle q_{i j}\right\rangle$. (The divisible hull is the injective hull within the category of abelian groups.) In view of our hypotheses, $\left\langle q_{i j}\right\rangle$ contains no elements of order 2 , whence the same holds for $D$. Now each $q_{i j}$ has a unique square root in $D$, say $p_{i j}$, and it follows from the uniqueness of the choices that $\left(p_{i j}\right)$ is a multiplicatively antisymmetric matrix over $k$. Thus, the rule

$$
c(\alpha, \beta)=\prod_{i, j=1}^{n} p_{i j}^{\alpha_{i} \beta_{j}}
$$


defines an alternating bicharacter $c$ on $\Gamma$ such that $c^{2}=\sigma$. For any $\alpha, \beta \in \Gamma$ such that $\sigma(\alpha, \beta)=1$, we have $c(\alpha, \beta)^{2}=1$ and therefore $c(\alpha, \beta)=1$, because $D$ has no elements of order 2 .

4.3. Fix an alternating bicharacter $c$ as in (4.2). Then $c$ is a 2-cocycle on $\Gamma$, and

$$
c(\alpha, \beta) c(\beta, \alpha)^{-1}=c(\alpha, \beta)^{2}=\sigma(\alpha, \beta)
$$

for $\alpha, \beta \in \Gamma$. As in (3.3), we write $k^{c} \Gamma$ in terms of a basis $\left\{x_{\alpha} \mid \alpha \in \Gamma\right\}$ such that $x_{\alpha} x_{\beta}=c(\alpha, \beta) x_{\alpha+\beta}$ for $\alpha, \beta \in \Gamma$. Thus $A=k^{c} \Gamma^{+}$equals the subspace of $k^{c} \Gamma$ spanned by the $x_{\alpha}$ for $\alpha \in \Gamma^{+}$.

Similarly, we write the group algebra $k \Gamma$ in terms of a basis $\left\{y_{\alpha} \mid \alpha \in \Gamma\right\}$, with $y_{\alpha} y_{\beta}=y_{\alpha+\beta}$ for $\alpha, \beta \in \Gamma$, and we identify the semigroup algebra $R:=k \Gamma^{+}$with the subspace of $k \Gamma$ spanned by the $y_{\alpha}$ for $\alpha \in \Gamma^{+}$. We also view $R$ as a polynomial ring $k\left[y_{1}, \ldots, y_{n}\right]$, as in Section 2 , where $y_{i}=y_{\epsilon_{i}}$ and $\epsilon_{i}$ is the $i$-th standard basis element of $\Gamma$.

4.4. Following (3.4), set $H=\operatorname{Hom}\left(\Gamma, k^{\times}\right)$and write the application of $H$ to $\Gamma$ in terms of a pairing $\langle-,-\rangle: H \times \Gamma \rightarrow k^{\times}$. We again have actions of $H$ on $k^{c} \Gamma$ and $k \Gamma$ via $k$-algebra automorphisms such that $h . x_{\alpha}=\langle h, \alpha\rangle x_{\alpha}$ and $h . y_{\alpha}=\langle h, \alpha\rangle y_{\alpha}$, for $h \in H$ and $\alpha \in \Gamma$. The subalgebras $A$ and $R$ are stable under these actions.

4.5. Let $W$ denote the set of subsets of $\{1, \ldots, n\}$. For $w \in W$, let $\operatorname{spec}_{w} A$ be the set, equipped with the relative Zariski topology, of those prime ideals $P$ in $A$ such that

$$
P \cap\left\{x_{1}, \ldots, x_{n}\right\}=\left\{x_{i} \mid i \in w\right\} .
$$

Then $\operatorname{spec} A$ is the disjoint union of the $\operatorname{sets} \operatorname{spec}_{w} A$. Likewise, $\operatorname{prim} A$ is the disjoint union of the sets $\operatorname{prim}_{w} A=\operatorname{prim} A \cap \operatorname{spec}_{w} A$, each of which is also endowed with the relative topology. Note that the $\operatorname{sets} \operatorname{spec}_{w} A$ and $\operatorname{prim}_{w} A$ are invariant under the action of $H$. Also, it was proved in [6, 2.3], for $w \in W$, that the set $\operatorname{prim}_{w} A$ coincides with the set of maximal members of $\operatorname{spec}_{w} A$. Define $\operatorname{spec}_{w} R$ and $\max _{w} R$ similarly, as in (2.1) and (2.5). Recall that $\max _{w} R$ equals the set of maximal elements of $\operatorname{spec}_{w} R$.

It is convenient to label the localizations

$$
A_{w}=\left(A /\left\langle x_{i} \mid i \in w\right\rangle\right)\left[x_{j}^{-1} \mid j \notin w\right] \quad \text { and } \quad R_{w}=\left(R /\left\langle y_{i} \mid i \in w\right\rangle\right)\left[y_{j}^{-1} \mid j \notin w\right] \text {, }
$$

and to identify $A_{w}$ (respectively, $R_{w}$ ) with the $k$-subalgebra of $k^{c} \Gamma$ (respectively, $k \Gamma$ ) spanned by the $x_{\alpha}$ (respectively, $y_{\alpha}$ ) for those $\alpha \in \Gamma$ such that $\alpha_{i}=0$ for $i \in w$. In view of the preceding paragraph and 7. 9.20], for example, we see that localization induces $H$-equivariant homeomorphisms

$$
\begin{aligned}
& \operatorname{spec}_{w} A \longrightarrow \operatorname{spec} A_{w}, \quad \operatorname{spec}_{w} R \longrightarrow \operatorname{spec} R_{w}, \\
& \operatorname{prim}_{w} A \longrightarrow \max A_{w}, \quad \max _{w} R \longrightarrow \max R_{w} \text {. }
\end{aligned}
$$

4.6. As in (3.5), we have an $H$-equivariant $k$-linear isomorphism $\Phi=\Phi_{c}: k^{c} \Gamma \rightarrow$ $k \Gamma$ such that $\Phi\left(x_{\alpha}\right)=y_{\alpha}$ for $\alpha \in \Gamma$. This map restricts to an $H$-equivariant $k$-linear isomorphism from $A$ onto $R$. Note that $\Phi$ also restricts to $H$-equivariant $k$-linear isomorphisms $\Phi_{w}: A_{w} \rightarrow R_{w}$ for $w \in W$.

4.7. Retain $\epsilon_{1}, \ldots, \epsilon_{n}$ as the standard basis for $\Gamma$, and fix $w \in W$. Let $\Gamma_{w}$ be the subgroup of $\Gamma$ generated by $\left\{\epsilon_{i} \mid i \notin w\right\}$, and let $\sigma_{w}$ and $c_{w}$ denote the restrictions of $\sigma$ and $c$ to $\Gamma_{w}$. With this notation, the identifications made in (4.5) are $A_{w}=k^{c_{w}} \Gamma_{w}$ and $R_{w}=k \Gamma_{w}$. 
4.8. Set $S_{w}=\operatorname{rad}\left(\sigma_{w}\right)=\left\{\alpha \in \Gamma_{w} \mid \sigma(\alpha,-) \equiv 1\right.$ on $\left.\Gamma_{w}\right\}$, for $w \in W$. As in (3.7), set

$$
S_{w}^{\perp}=\left\{h \in H \mid\langle h, \alpha\rangle=1 \text { for } \alpha \in S_{w}\right\}
$$

and

$$
\Gamma_{w}^{\perp}=\left\{h \in H \mid\langle h, \alpha\rangle=1 \text { for } \alpha \in \Gamma_{w}\right\} .
$$

Because $\sigma \equiv 1$ on $S_{w} \times \Gamma_{w}$, it follows from (4.2) that $c \equiv 1$ on $S_{w} \times \Gamma_{w}$.

Lemma. Let $v \subseteq w$ in $W$.

(a) $S_{w}^{\perp} \subseteq S_{v}^{\perp} \Gamma_{w}^{\perp}$.

(b) $\left(P: S_{v}^{\perp}\right) \subseteq\left(P: S_{w}^{\perp}\right)$ for all $P \in \operatorname{spec}_{w} R$.

Proof. (a) Observe that $\Gamma_{w} \subseteq \Gamma_{v}$ and that $S_{v} \cap \Gamma_{w} \subseteq S_{w}$. Any $h \in S_{w}^{\perp}$ is a homomorphism $\Gamma \rightarrow k^{\times}$such that ker $h \supseteq S_{w} \supseteq S_{v} \cap \Gamma_{w}$. Hence, $\left.h\right|_{\Gamma_{w}}$ extends to a homomorphism $h_{1}: \Gamma_{w}+S_{v} \rightarrow k^{\times}$such that $S_{v} \subseteq \operatorname{ker} h_{1}$. Since $k^{\times}$is divisible, $h_{1}$ extends to a homomorphism $h_{2}: \Gamma \rightarrow k^{\times}$. Now $h_{2}$ is a homomorphism in $H$ such that $h_{2}=h$ on $\Gamma_{w}$ and $h_{2}=1$ on $S_{v}$. Thus $h_{2} \in S_{v}^{\perp}$ and $h_{2}^{-1} h \in \Gamma_{w}^{\perp}$, whence $h \in S_{v}^{\perp} \Gamma_{w}^{\perp}$, as desired.

(b) Observe that $\Gamma_{w}^{\perp}$ acts trivially on $R /\left\langle y_{i} \mid i \in w\right\rangle$. Hence, $P$ is stable under $\Gamma_{w}^{\perp}$, and so $\left(P: S_{v}^{\perp}\right)=\left(P: S_{v}^{\perp} \Gamma_{w}^{\perp}\right)$. In view of part (a), $\left(P: S_{v}^{\perp} \Gamma_{w}^{\perp}\right) \subseteq\left(P: S_{w}^{\perp}\right)$, and we are done.

4.9. Set $\mathcal{G}=\left(S_{w}^{\perp}\right)_{w \in W}$, and note by (4.8) that $\mathcal{G}$ satisfies the hypotheses in (2.2), as applied to $R$. Define $\mathcal{G}$-spec $R$ as in $(2.2)$, and define $(P: \mathcal{G})$ to be $\left(P: S_{w}^{\perp}\right)$ for $P \in \operatorname{spec}_{w} R$, as in (2.3). Apply the Zariski topology to $\mathcal{G}$-spec $R$, following (2.4). We may also use $(2.4)$ to conclude that the assignment $P \mapsto(P: \mathcal{G})$ is a topological quotient map from $\operatorname{spec} R$ onto $\mathcal{G}$-spec $R$.

Next, define $\mathcal{G}-\max R$ as in (2.5). It follows from (2.6), for each $w \in W$, that $\mathcal{G}-\max _{w} R$ is the set of maximal members of $\mathcal{G}-\operatorname{spec}_{w} R$. From (2.5) it follows that the assignment $M \mapsto(M: \mathcal{G})$, for $M \in \max R$, produces an $H$-equivariant topological quotient map from $\max R$ onto $\mathcal{G}$-max $R$. Moreover, by $(2.7)$, the fibers in $\max R$ over points in $\mathcal{G}-\max _{w} R$ are precisely the $S_{w}^{\perp}$-orbits in $\max _{w} R$.

It remains to relate $\operatorname{spec} A$ to $\mathcal{G}-\operatorname{spec} R$ and $\operatorname{prim} A$ to $\mathcal{G}$-max $R$.

4.10 Lemma. The set function $I \mapsto \Phi(I)$, for ideals $I$ of $R$, produces an $H$ equivariant homeomorphism from spec $A$ onto $\mathcal{G}$-spec $R$ that restricts to a homeomorphism from $\operatorname{prim} A$ onto $\mathcal{G}$ - $\max R$.

Proof. Given $w \in W$, there exists an $H$-equivariant commutative diagram

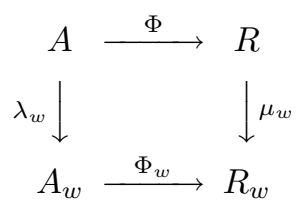

where $\lambda_{w}$ and $\mu_{w}$ are the respective localization maps. Let $Q \in \mathcal{G}-\operatorname{spec}_{w} R$, and write $Q=\left(P: S_{w}^{\perp}\right)$ for some $P \in \operatorname{spec}_{w} R$. Then $P=\mu_{w}^{-1}\left(P^{\prime}\right)$ for some $P^{\prime} \in$ $\operatorname{spec} R_{w}$; the ideal $Q^{\prime}=\left(P^{\prime}: S_{w}^{\perp}\right)$ lies in $S_{w}^{\perp-\operatorname{spec}} R_{w}$; and $Q=\mu_{w}^{-1}\left(Q^{\prime}\right)$. By (3.9), $\Phi_{w}^{-1}\left(Q^{\prime}\right) \in \operatorname{spec} A_{w}$, and so $\lambda_{w}^{-1} \Phi_{w}^{-1}\left(Q^{\prime}\right) \in \operatorname{spec}_{w} A$. Since $\lambda_{w}^{-1} \Phi_{w}^{-1}\left(Q^{\prime}\right)=$ $\Phi^{-1} \mu_{w}^{-1}\left(Q^{\prime}\right)=\Phi^{-1}(Q)$, we thus see that the set $\Phi^{-1}(Q)$ is a prime ideal of $A$, lying in $\operatorname{spec}_{w} A$. Similarly, $\Phi^{-1}(M) \in \operatorname{prim}_{w} A$ for all $M \in \mathcal{G}-\max _{w} R$. 
Since $\Phi$ and $\Phi_{w}$ are bijections, similar arguments show that $\Phi(Q) \in \mathcal{G}-$ spec $_{w} R$, for all $Q \in \operatorname{spec}_{w} A$, and $\Phi(P) \in \mathcal{G}-\max _{w} R$, for all $P \in \operatorname{prim}_{w} A$.

The results above, taken over all $w \in W$, show that the set maps $I \mapsto \Phi(I)$ and $J \mapsto \Phi^{-1}(J)$ yield pairs of mutually inverse bijections

$$
\operatorname{spec} A \longleftrightarrow \mathcal{G} \text {-spec } R \quad \text { and } \quad \operatorname{prim} A \longleftrightarrow \mathcal{G}-\max R .
$$

These maps are $H$-equivariant because $\Phi$ and $\Phi^{-1}$ are $H$-equivariant. Finally, note that the topologies on all of the spaces considered in this section can be defined using subsets of $A$ and $R$ instead of ideals - for instance, the closed subsets of spec $A$ are precisely those of the form $\{Q \in \operatorname{spec} A \mid Q \supseteq X\}$, for arbitrary subsets $X \subseteq A$. It therefore follows that the above maps are homeomorphisms, as desired.

Combining (4.9) and (4.10), we obtain the main theorem of the paper, which we state as follows, using the notation developed in (4.2)-(4.8).

4.11 Theorem. Suppose that $k$ is an algebraically closed field, that $\mathbf{q}=\left(q_{i j}\right)$ is a multiplicatively antisymmetric $n \times n$ matrix over $k$, that $A=\mathcal{O}_{\mathbf{q}}\left(k^{n}\right)$, and that $R=\mathcal{O}\left(k^{n}\right)$. Further assume that $-1 \notin\left\langle q_{i j}\right\rangle$ or that char $k=2$. Let $H=\left(k^{\times}\right)^{n}$ act by $k$-algebra automorphisms on $A$ and on $R$ in the standard manner. Then there exist $H$-equivariant topological quotient maps

$$
\operatorname{spec} R \rightarrow \operatorname{spec} A \quad \text { and } \quad \max R \rightarrow \operatorname{prim} A
$$

given by $P \mapsto \Phi^{-1}\left(P: S_{w}^{\perp}\right)$, for $P \in \operatorname{spec}_{w} R$. The fibers of the second map, over points in $\operatorname{prim}_{w} A$, consist precisely of the $S_{w}^{\perp}$-orbits within $\max _{w} R$.

\section{Some EXAmples}

We illustrate (4.11) by calculating the explicit form of the maps from $\max R$ to $\operatorname{prim} A$ in the cases of the standard single parameter quantum affine spaces, and we comment on some more general cases related to bilinear forms and Poisson brackets. Let $k, \mathbf{q}, A$, and $R$ be as in (4.11), and let

$$
\Psi: \max R \longrightarrow \operatorname{prim} A
$$

denote the topological quotient map given by the theorem therein. Composing $\Psi$ with the natural isomorphism of affine $n$-space onto $\max R$, we obtain a similar topological quotient map

$$
\psi: k^{n} \longrightarrow \operatorname{prim} A
$$

For simplicity of notation, we describe $\psi$ rather than $\Psi$.

5.1. First, let $A$ be the standard one-parameter quantization $\mathcal{O}_{q}\left(k^{n}\right)$, for some $q \in k^{\times}$. Then $A$ is generated by $x_{1}, \ldots, x_{n}$ such that $x_{i} x_{j}=q x_{j} x_{i}$ for all $i<j$. The alternating bicharacter $\sigma$ as in (4.1) can be expressed by

$$
\sigma(\alpha, \beta)=q^{b(\alpha, \beta)},
$$

where $b: \Gamma \times \Gamma \rightarrow \mathbb{Z}$ is the alternating bilinear form

$$
b(\alpha, \beta)=\sum_{i<j} \alpha_{i} \beta_{j}-\sum_{i>j} \alpha_{i} \beta_{j} .
$$


To complete the hypotheses of (4.11), we must assume that $q$ is either not a root of unity or an odd root of unity. In either case, $q$ has a square root $p \in k^{\times}$such that $-1 \notin\langle p\rangle$ (if char $k \neq 2$ ), and the rule

$$
c(\alpha, \beta)=p^{b(\alpha, \beta)}
$$

defines an alternating bicharacter $c$ on $\Gamma$ satisfying the conclusions of (4.2). Hence, we can identify $A$ with $k^{c} \Gamma^{+}$for this $c$.

5.2. Continue with (5.1), and suppose that $q$ is not a root of unity. We calculate $\psi(\boldsymbol{\lambda})$ for points $\boldsymbol{\lambda}=\left(\lambda_{1}, \ldots, \lambda_{n}\right)$ in $k^{n}$. This depends on the position of $\boldsymbol{\lambda}$, with respect to the stratification of $k^{n}$ corresponding to the subsets $\max _{w} R$. Set

$$
\left(k^{n}\right)_{w}=\left\{\boldsymbol{\lambda} \in k^{n} \mid \lambda_{i}=0 \text { for } i \in w \text { and } \lambda_{j} \neq 0 \text { for } j \notin w\right\}
$$

for $w \in W$.

If $n-|w|$ is even, we calculate that $S_{w}=0$. In this case,

$$
\psi(\boldsymbol{\lambda})=\left\langle x_{i} \mid i \in w\right\rangle
$$

for all $\boldsymbol{\lambda} \in\left(k^{n}\right)_{w}$.

Now suppose that $n-|w|$ is odd. List the elements of the complement of $w$ in ascending order, say

$$
\{1, \ldots, n\} \backslash w=\left\{w_{1}<w_{2}<\cdots<w_{2 m+1}\right\},
$$

and then set

$$
w_{+}=\left\{w_{1}, w_{3}, \ldots, w_{2 m+1}\right\} \quad \text { and } \quad w_{-}=\left\{w_{2}, w_{4}, \ldots, w_{2 m}\right\} .
$$

Finally, write $\gamma_{ \pm}(w)=\sum_{i \in w_{ \pm}} \epsilon_{i} \in \Gamma$, where $\epsilon_{1}, \ldots, \epsilon_{n}$ is, as usual, the standard basis for $\Gamma$. We calculate that $S_{w}=\mathbb{Z}\left(\gamma_{+}(w)-\gamma_{-}(w)\right)$, and that

$$
\psi(\boldsymbol{\lambda})=\left\langle x_{i} \mid i \in w\right\rangle+\left\langle\left(\prod_{j \in w_{-}} \lambda_{j}\right) x_{\gamma_{+}(w)}-\left(\prod_{j \in w_{+}} \lambda_{j}\right) x_{\gamma_{-}(w)}\right\rangle
$$

for $\boldsymbol{\lambda} \in\left(k^{n}\right)_{w}$.

If one wishes to write the last formula in terms of ordinary monomials in the $x_{i}$, additional scalar factors are introduced. For instance, suppose that $n=3$ and $w=\varnothing$, so that $w_{+}=\{1,3\}$ and $w_{-}=\{2\}$. Since $x_{1} x_{3}=c\left(\epsilon_{1}, \epsilon_{3}\right) x_{(1,0,1)}=p x_{\gamma_{+}(w)}$, we have

$$
\psi\left(\lambda_{1}, \lambda_{2}, \lambda_{3}\right)=\left\langle p^{-1} \lambda_{2} x_{1} x_{3}-\lambda_{1} \lambda_{3} x_{2}\right\rangle
$$

for $\left(\lambda_{1}, \lambda_{2}, \lambda_{3}\right) \in\left(k^{3}\right)_{w}=\left(k^{\times}\right)^{3}$.

5.3. Continue with (5.1), but suppose now that $q$ is a primitive $t$-th root of unity, for some odd $t>1$. In this case, the correct choice for $p$ is $q^{(t+1) / 2}$. The form of $\psi(\boldsymbol{\lambda})$ for $\boldsymbol{\lambda} \in\left(k^{n}\right)_{w}$ again depends on the parity of $n-|w|$.

If $n-|w|$ is even, we calculate that $S_{w}=t \Gamma_{w}$, and that

$$
\psi(\boldsymbol{\lambda})=\left\langle x_{i} \mid i \in w\right\rangle+\left\langle x_{j}^{t}-\lambda_{j}^{t} \mid j \notin w\right\rangle
$$

for $\boldsymbol{\lambda} \in\left(k^{n}\right)_{w}$.

If $n-|w|$ is odd, we calculate that $S_{w}=t \Gamma_{w}+\mathbb{Z}\left(\gamma_{+}(w)-\gamma_{-}(w)\right)$, and that

$$
\psi(\boldsymbol{\lambda})=\left\langle x_{i} \mid i \in w\right\rangle+\left\langle x_{j}^{t}-\lambda_{j}^{t} \mid j \notin w\right\rangle+\left\langle\left(\prod_{j \in w_{-}} \lambda_{j}\right) x_{\gamma_{+}(w)}-\left(\prod_{j \in w_{+}} \lambda_{j}\right) x_{\gamma_{-}(w)}\right\rangle
$$

for $\boldsymbol{\lambda} \in\left(k^{n}\right)_{w}$. 
5.4. Let $b: \Gamma \times \Gamma \rightarrow \mathbb{Z}$ be an arbitrary alternating bilinear form, let $q \in k^{\times}$be a non-root of unity, and set $q_{i j}=q^{b\left(\epsilon_{i}, \epsilon_{j}\right)}$ for all $i, j$. The alternating bicharacter $\sigma$ associated with $A$ as in (4.1) then takes the form $\sigma(\alpha, \beta)=q^{b(\alpha, \beta)}$. To obtain a bicharacter $c$ as in (4.2), take a square root $p$ for $q$, and define $c(\alpha, \beta)=p^{b(\alpha, \beta)}$. Observe that since $q$ is not a root of unity, $S_{w}=\operatorname{rad}\left(\left.\sigma\right|_{\Gamma_{w}}\right)=\operatorname{rad}\left(\left.b\right|_{\Gamma_{w}}\right)$ for $w \in W$.

Now suppose that char $k=0$. There is a Poisson bracket on $R$ such that $\left\{y_{\alpha}, y_{\beta}\right\}=b(\alpha, \beta) y_{\alpha} y_{\beta}$ for $\alpha, \beta \in \Gamma$. The analysis in [15, 1.4] (with $\mathbb{C}$ replaced by $k$ ) shows that the $S_{w}^{\perp}$-orbits in $\left(k^{n}\right)_{w}$ coincide with the minimal Poisson subvarieties relative to the Poisson structure just defined. Therefore the fibers of $\psi$ in this case coincide with the minimal Poisson subvarieties of $k^{n}$.

When $k=\mathbb{C}$, these minimal Poisson subvarieties are not necessarily the same as the symplectic leaves in $k^{n}$. See [15, 1.4] for necessary and sufficient conditions.

5.5. The relation $\sigma=q^{b}$ in (5.4) is stronger than necessary for the analysis given - it would suffice to have an alternating bilinear form $b: \Gamma \times \Gamma \rightarrow k$ such that $\operatorname{rad}\left(\left.\sigma\right|_{\Gamma_{w}}\right)=\operatorname{rad}\left(\left.b\right|_{\Gamma_{w}}\right)$ for all $w \in W$. To find further examples where such $b$ exist, and some situations in which the fibers of a map analogous to $\psi$ coincide with symplectic leaves, see [15, Section 3].

5.6. To test whether the hypothesis $-1 \notin\left\langle q_{i j}\right\rangle$ in (4.11) is necessary, the natural examples to investigate are the algebras $\mathcal{O}_{-1}\left(k^{n}\right)$ in characteristic different from 2. Our method works precisely when $\mathcal{O}_{-1}\left(k^{n}\right)$ can be expressed in the form $k^{c} \Gamma^{+}$for some 2-cocycle $c$ such that $c \equiv 1$ on $S_{w} \times \Gamma_{w}$ for all $w \in W$. It is easy to find such a $c$ in case $n=2$, and one also exists in case $n=3$. However, it can be shown that no such $c$ exists when $n \geq 4$. Thus, our method cannot handle $\mathcal{O}_{\mathbf{q}}\left(k^{n}\right)$ for arbitrary $\mathbf{q}$ and $n$. We leave it as an open question whether $\operatorname{prim} \mathcal{O}_{\mathbf{q}}\left(k^{n}\right)$ is always an $H$-equivariant topological quotient of $\max \mathcal{O}\left(k^{n}\right)$.

\section{Twists of Graded Commutative algebras}

To conclude the paper, we apply the main theorem (4.11) to a class of twists of finitely generated commutative graded algebras. Assume throughout that $k$ is a field, that $R$ is a $k$-algebra graded by an abelian group $G$ (which we write additively), and that $c: G \times G \rightarrow k^{\times}$is a 2-cocycle.

6.1. There exists a $G$-graded $k$-algebra $R^{\prime}$ equipped with a $G$-graded $k$-linear isomorphism

$$
\begin{aligned}
& R \longrightarrow R^{\prime}, \\
& r \longmapsto r^{\prime},
\end{aligned}
$$

such that, for all $\alpha, \beta \in G$, the multiplication of arbitrary homogeneous elements $r^{\prime} \in R_{\alpha}^{\prime}$ and $s^{\prime} \in R_{\beta}^{\prime}$ is given by the rule $r^{\prime} s^{\prime}=c(\alpha, \beta)(r s)^{\prime}$. Up to a $G$-graded $k$-algebra isomorphism, $R^{\prime}$ depends only on the cohomology class of $c$ [2, p. 888]. We call $R^{\prime}$ the twist of $R$ by $c$ (cf. [2, Section 3]), and we refer to the above function $R \rightarrow R^{\prime}$ as the twist map (associated to $c$ ). If $U$ is a subset of $R$, we will use $U^{\prime}$ to denote the image of $U$ under the twist map.

Note, for computational purposes, that if $I$ and $J$ are $(G$-)homogeneous ideals of $R$, then $I^{\prime} J^{\prime}=(I J)^{\prime}$.

6.2. Recall that a proper homogeneous ideal $K$, in $R$ or $R^{\prime}$, is graded-prime provided it contains no product $I J$ of homogeneous ideals $I$ and $J$ not contained in $K$. Every ideal of $R$ or $R^{\prime}$ contains a unique maximum homogeneous ideal, and 
an argument similar to (1.3) demonstrates that the maximum homogeneous ideal within a prime ideal is graded-prime.

Lemma. Suppose that $G$ is torsionfree and that $R$ is noetherian. If $P_{1}, \ldots, P_{m}$ are the minimal prime ideals of $R$, then $P_{1}, \ldots, P_{m}$ are homogeneous, and $P_{1}^{\prime}, \ldots, P_{m}^{\prime}$ are the minimal prime ideals of $R^{\prime}$.

Proof. Let $P_{i}^{\circ}$ denote the largest homogeneous ideal contained in $P_{i}$. Since $G$ is torsionfree, the graded-prime ideal $P_{i}^{\circ}$ must actually be a prime ideal, by [1] Corollary 3.3]. Hence, $P_{i}=P_{i}^{\circ}$ by minimality.

Since the twist map preserves homogeneous ideals and their products, it follows that $P_{i}^{\prime}$ is a graded-prime ideal of $R^{\prime}$. A second application of [1, Corollary 3.3] then shows that $P_{i}^{\prime}$ is a prime ideal of $R^{\prime}$. Now some product of the $P_{i}$ is equal to 0 in $R$, and by homogeneity the corresponding product of the $P_{i}^{\prime}$ must be 0 in $R^{\prime}$. It follows that every minimal prime ideal of $R^{\prime}$ must occur among the $P_{i}^{\prime}$.

Let $Q_{1}^{\prime}, \ldots, Q_{n}^{\prime}$ be the minimal prime ideals of $R^{\prime}$, and denote their respective preimages under the twist map by $Q_{1}, \ldots, Q_{n}$. Arguing as above, we see that the $Q_{j}$ are prime ideals of $R$, and that all minimal prime ideals of $R$ occur among the $Q_{j}$. The lemma follows.

6.3 Theorem. Let $k$ be an algebraically closed field, let $R$ be a commutative affine $k$-algebra graded by a torsionfree abelian group $G$, and let $A$ be the twist of $R$ by a 2-cocycle c : $G \times G \rightarrow k^{\times}$. Assume either that -1 is not in the subgroup of $k^{\times}$ generated by the image of $c$, or that char $k=2$. Then there exist topological quotient maps

$$
\operatorname{spec} R \rightarrow \operatorname{spec} A \quad \text { and } \quad \max R \rightarrow \operatorname{prim} A
$$

Proof. Since $R$ is affine, its support is contained in a finitely generated subgroup $G^{\mathrm{fg}} \subseteq G$. Then $R$ is also graded by $G^{\mathrm{fg}}$, and $A$ is equal to the twist of $R$ by $\left.c\right|_{G^{\mathrm{fg}}}$. Hence, there is no loss of generality in assuming that $G$ is finitely generated. Thus $G$ is now a free abelian group of finite rank.

Set $\sigma(\alpha, \beta)=c(\alpha, \beta) c(\beta, \alpha)^{-1}$, for $\alpha, \beta \in G$. By [2, Proposition 1(ii), p. 888], $\sigma$ is an alternating bicharacter on $G$. Since either $-1 \notin\langle\operatorname{im} \sigma\rangle$ or char $k=2$, it follows from (4.2) that there exists an alternating bicharacter $d$ on $G$ with the following properties: $d^{2}=\sigma$, and $d(\alpha, \beta)=1$ whenever $\sigma(\alpha, \beta)=1$. By [2, Proposition 1(i), p. 888], $d$ and $c$ are in the same cohomology class, and hence $A$ can also be written as the twist of $R$ by $d$. Thus, there is no loss of generality in assuming that $c$ is an alternating bicharacter and that $c(\alpha, \beta)=1$ whenever $c(\alpha, \beta)^{2}=1$.

The inverse of the twist map is a $G$-graded $k$-linear isomorphism $\Phi: A \rightarrow R$ such that $\Phi(a b)=c(\alpha, \beta) \Phi(a) \Phi(b)$ for $a \in A_{\alpha}$ and $b \in A_{\beta}$. Choose homogeneous elements $r_{1}, \ldots, r_{n}$ generating $R$ as a $k$-algebra, and let $\delta_{1}, \ldots, \delta_{n} \in G$ be the degrees of these elements.

Set $\Gamma=\mathbb{Z}^{n}$ and $\Gamma^{+}=\left(\mathbb{Z}^{+}\right)^{n}$, and let $\rho: \Gamma \rightarrow G$ be the group homomorphism given by

$$
\rho\left(\alpha_{1}, \ldots, \alpha_{n}\right)=\alpha_{1} \delta_{1}+\cdots+\alpha_{n} \delta_{n} .
$$

Set $\widetilde{c}(\alpha, \beta)=c(\rho(\alpha), \rho(\beta))$ for $\alpha, \beta \in \Gamma$. Then $\widetilde{c}$ is an alternating bicharacter on $\Gamma$ such that $\widetilde{c}(\alpha, \beta)=1$ whenever $\widetilde{c}(\alpha, \beta)^{2}=1$. Now set $r^{\alpha}=r_{1}^{\alpha_{1}} r_{2}^{\alpha_{2}} \cdots r_{n}^{\alpha_{n}} \in R_{\rho(\alpha)}$ and $a_{\alpha}=\Phi^{-1}\left(r^{\alpha}\right) \in A_{\rho(\alpha)}$ for $\alpha \in \Gamma^{+}$. Since $R$ is commutative, $r^{\alpha} r^{\beta}=r^{\alpha+\beta}$ for 
$\alpha, \beta \in \Gamma^{+}$, whence

$$
\Phi\left(a_{\alpha} a_{\beta}\right)=c(\rho(\alpha), \rho(\beta)) r^{\alpha} r^{\beta}=\widetilde{c}(\alpha, \beta) r^{\alpha+\beta}=\widetilde{c}(\alpha, \beta) \Phi\left(a_{\alpha+\beta}\right),
$$

and so $a_{\alpha} a_{\beta}=\widetilde{c}(\alpha, \beta) a_{\alpha+\beta}$ for $\alpha, \beta \in \Gamma^{+}$.

Now set $\widetilde{R}=k \Gamma^{+}$and $\widetilde{A}=k^{\widetilde{c}} \Gamma^{+}$. Write $\widetilde{R}$ in terms of a $k$-basis $\left\{y_{\alpha} \mid \alpha \in \Gamma^{+}\right\}$ such that $y_{\alpha} y_{\beta}=y_{\alpha+\beta}$ for $\alpha, \beta \in \Gamma^{+}$, and analogously write $\widetilde{A}$ in terms of a $k$-basis $\left\{x_{\alpha} \mid \alpha \in \Gamma^{+}\right\}$such that $x_{\alpha} x_{\beta}=\widetilde{c}(\alpha, \beta) x_{\alpha+\beta}$ for $\alpha, \beta \in \Gamma^{+}$. Let $\widetilde{\Phi}: \widetilde{A} \rightarrow \widetilde{R}$ be the $k$-linear isomorphism such that $\widetilde{\Phi}\left(x_{\alpha}\right)=y_{\alpha}$ for $\alpha \in \Gamma^{+}$. There are $k$-algebra epimorphisms $f: \widetilde{A} \rightarrow A$ and $g: \widetilde{R} \rightarrow R$ such that $f\left(x_{\alpha}\right)=a_{\alpha}$ and $g\left(y_{\alpha}\right)=r^{\alpha}$ for $\alpha \in \Gamma^{+}$. We now obtain the following commutative diagram:

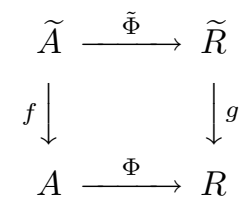

Thus if $I=\operatorname{ker} f$, then $\widetilde{\Phi}(I)=\operatorname{ker} g$.

Define $\mathcal{G}$ as in (4.9), with $\widetilde{c}, \widetilde{R}, \widetilde{A}$ playing the roles of $c, R, A$. By (4.11), the rule $P \mapsto \widetilde{\Phi}^{-1}(P: \mathcal{G})$ gives topological quotient maps

$$
\phi_{s}: \operatorname{spec} \widetilde{R} \rightarrow \operatorname{spec} \widetilde{A} \quad \text { and } \quad \phi_{m}: \max \widetilde{R} \rightarrow \operatorname{prim} \widetilde{A} .
$$

These maps restrict to topological quotient maps

$$
\begin{gathered}
\phi_{s}^{-1} V(I) \rightarrow V(I) \approx \operatorname{spec} A, \\
\phi_{m}^{-1}(V(I) \cap \operatorname{prim} \widetilde{A}) \rightarrow V(I) \cap \operatorname{prim} \widetilde{A} \approx \operatorname{prim} A .
\end{gathered}
$$

To finish the proof of the theorem, it suffices to show that $\phi_{s}^{-1} V(I)=V(\widetilde{\Phi}(I))$. The inclusion $\subseteq$ is easy: If $P \in \phi_{s}^{-1} V(I)$, then $\widetilde{\Phi}^{-1}(P: \mathcal{G}) \supseteq I$, whence $P \supseteq$ $(P: \mathcal{G}) \supseteq \widetilde{\Phi}(I)$.

Let $P_{1}, \ldots, P_{m}$ be the prime ideals of $\widetilde{A}$ minimal over $I$. In view of $(6.2)$, the sets $\widetilde{\Phi}\left(P_{1}\right), \ldots, \widetilde{\Phi}\left(P_{m}\right)$ are the prime ideals of $\widetilde{R}$ minimal over $\widetilde{\Phi}(I)$. By (4.10), each $\widetilde{\Phi}\left(P_{j}\right) \in \mathcal{G}$-spec $\widetilde{R}$. Given any $P \in V(\widetilde{\Phi}(I))$, we have $P \supseteq \widetilde{\Phi}\left(P_{j}\right)$ for some $j$. Since $\widetilde{\Phi}\left(P_{j}\right)$ is a $\mathcal{G}$-prime ideal, $(P: \mathcal{G}) \supseteq \widetilde{\Phi}\left(P_{j}\right) \supseteq \widetilde{\Phi}(I)$, and thus $\phi_{s}(P) \in V(I)$. Therefore $\phi_{s}^{-1} V(I)=V(\widetilde{\Phi}(I))$, as desired.

6.4 Remark. Retain the notation in the statement of the preceding theorem, and let $r_{1}, \ldots, r_{n}$ be homogeneous generators for $R$. As seen in the proof, we may assume without loss of generality that $G=\mathbb{Z}^{n}$ and that $c$ is an alternating bicharacter on $\mathbb{Z}^{n}$. In particular, it can be shown that the topological quotient maps in (6.3) are equivariant with respect to suitable actions by a subgroup of $\left(k^{\times}\right)^{n}$; details are left to the interested reader.

\section{Note ADDED IN PROOF}

A more precise version of (6.3), giving explicit formulas for the topological quotient maps and exhibiting equivariance with respect to actions of the group $\operatorname{Hom}\left(G, k^{\times}\right)$on $R$ and $A$, is given in the first author's paper (Quantized primitive ideal spaces as quotients of affine algebraic varieties, xxx.lanl.gov/abs/math.QA/ $9912110)$. 


\section{REFERENCES}

1. G. Abrams and J. Haefner, Primeness conditions for group graded rings, in Ring Theory, Proc. Biennial Ohio State - Denison Conf. 1992, World Scientific, Singapore, 1993, pp. 1-19. MR 96f:16050

2. M. Artin, W. Schelter, and J. Tate, Quantum deformations of $G L_{n}$, Communic. Pure Appl. Math. 44 (1991), 879-895. MR 92i:17014

3. K. A. Brown and K. R. Goodearl, Prime spectra of quantum semisimple groups, Trans. Amer. Math. Soc. 348 (1996), 2465-2502. MR 96i:17007

4. C. De Concini, V. Kac, and C. Procesi, Some remarkable degenerations of quantum groups, Comm. Math. Phys. 157 (1993), 405-427. MR 94i:17019

5. A. W. Goldie and G. O. Michler, Ore extensions and polycyclic group rings, J. London Math. Soc. (2) 9 (1974), 337-345. MR 50:9968

6. K. R. Goodearl and E. S. Letzter, Prime and primitive spectra of multiparameter quantum affine spaces, in Trends in Ring Theory. Proc. Miskolc Conf. 1996 (V. Dlab and L. Márki, eds.), Canad. Math. Soc. Conf. Proc. Series 22 Amer. Math. Soc., Providence, RI, 1998, pp. 39-58. MR 99h:16045

7. K. R. Goodearl and R. B. Warfield, Jr., An Introduction to Noncommutative Noetherian Rings, London Math. Soc. Student Texts 16, Cambridge Univ. Press, Cambridge, 1989. MR 91c:16001

8. T. J. Hodges and T. Levasseur, Primitive ideals of $\mathbf{C}_{q}[S L(3)]$, Commun. Math. Phys. 156 (1993), 581-605. MR 94k:17023

9. — Primitive ideals of $\mathbf{C}_{q}[S L(n)]$, J. Algebra 168 (1994), 455-468. MR 95i:16038

10. T. J. Hodges, T. Levasseur, and M. Toro, Algebraic structure of multi-parameter quantum groups, Advances in Math. 126 (1997), 52-92. MR 98e:17023

11. A. Joseph, On the prime and primitive spectra of the algebra of functions on a quantum group, J. Algebra 169 (1994), 441-511. MR 96b:17015

12. _ Quantum Groups and Their Primitive Ideals, Ergebnisse der Math. (3) 29, SpringerVerlag, Berlin, 1995. MR 96d:17015

13. J. C. McConnell and J. J. Pettit, Crossed products and multiplicative analogs of Weyl algebras, J. London Math. Soc. (2) 38 (1988), 47-55. MR 90c:16011

14. D. G. Northcott, Affine Sets and Affine Groups, London Math. Soc. Lecture Note Series 39, Cambridge Univ. Press, Cambridge, 1980. MR 82c:14002

15. M. Vancliff, Primitive and Poisson spectra of twists of polynomial rings, Algebras and Representation Theory 2 (1999), 269-285. CMP 2000:02

Department of Mathematics, University of California, Santa Barbara, California 93106

E-mail address: goodearl@math.ucsb.edu

Department of Mathematics, Texas A\&M University, College Station, Texas 77843

E-mail address: letzter@math.tamu.edu

Current address: Department of Mathematics, Temple University, Philadelphia, Pennsylvania 19122

E-mail address: letzter@math.temple.edu 